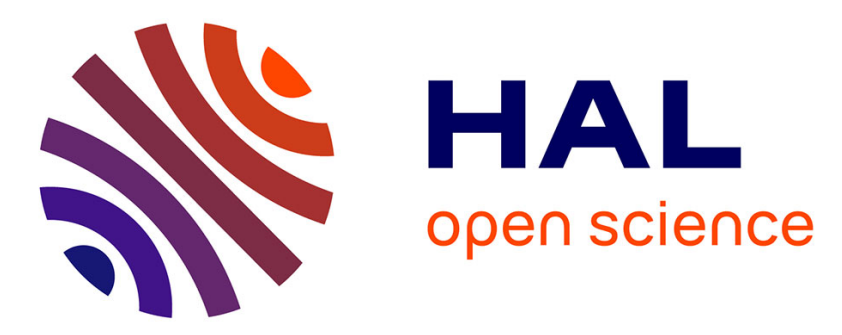

\title{
Investigation of the electron-acoustic phonon interaction via the deformation and piezoelectric potentials in AlN/GaN resonant tunneling nanostructures
}

I. Boyko, M. Petryk, J. Fraissard

\section{- To cite this version:}

I. Boyko, M. Petryk, J. Fraissard. Investigation of the electron-acoustic phonon interaction via the deformation and piezoelectric potentials in AlN/GaN resonant tunneling nanostructures. Superlattices and Microstructures, 2021, 156, pp.106928. 10.1016/j.spmi.2021.106928 . hal-03360121

\section{HAL Id: hal-03360121 \\ https: / hal.sorbonne-universite.fr/hal-03360121}

Submitted on 30 Sep 2021

HAL is a multi-disciplinary open access archive for the deposit and dissemination of scientific research documents, whether they are published or not. The documents may come from teaching and research institutions in France or abroad, or from public or private research centers.
L'archive ouverte pluridisciplinaire $\mathbf{H A L}$, est destinée au dépôt et à la diffusion de documents scientifiques de niveau recherche, publiés ou non, émanant des établissements d'enseignement et de recherche français ou étrangers, des laboratoires publics ou privés. 
Graphical Abstract

Investigation of the electron-acoustic phonon interaction via the deformation and piezoelectric potentials in AIN/GaN resonant tunneling nanostructures

I. Boyko,M. Petryk,J. Fraissard 


\section{Highlights}

Investigation of the electron-acoustic phonon interaction via the deformation and piezoelectric potentials in AIN/GaN resonant tunneling nanostructures

I. Boyko,M. Petryk,J. Fraissard

- Investigation of the electron-acoustic phonon interaction mechanisms in nitride resonant tunneling structures taking into account piezoelectric effect

- Electron-acoustic phonon interaction via the deformation potential in the presence of piezoelectric effect

- Electron-acoustic phonon interaction via the piezoelectric potential 


\title{
Investigation of the electron-acoustic phonon interaction via the deformation and piezoelectric potentials in AlN/GaN resonant tunneling nanostructures
}

\author{
I. Boyko ${ }^{a, *}$, M. Petryk ${ }^{a}$ and J. Fraissard ${ }^{b}$ \\ ${ }^{a}$ Ternopil Ivan Puluj National Technical University, Ruska str., 56, Ternopil 46001, Ukraine \\ ${ }^{b}$ Sorbonne Universités, ESPCI-LPEM, 10 rue Vauquelin, F-75231 Paris, France
}

\section{ARTICLE INFO}

\section{Keywords:}

acoustic phonon

electron-phonon interaction

piezoelectric effect

deformation potential

piezoelectric potential

Green's function

\begin{abstract}
A B S T R ACT
The analytical theory of acoustic phonons arising in the layers of a nitride-based nanostructure, taking into account the presence of the piezoelectric effect, was developed. Proposed theory is based on the first obtained exact solutions of the system consisting of equation of motion for a semiconductor medium and Maxwell equation. The theory of the electronic spectrum renormalized by their interaction with acoustic phonons via deformation and piezoelectric potentials was developed using the method of the temperature Green's function and the Dyson equation. Direct calculations of the electron level shifts and their decay rates are performed for geometric and physical parameters of the new experimentally created nanostructure for various temperature values.
\end{abstract}

\section{Introduction}

Semiconductor nitride compounds AlN, GaN, AlGaN and others are now widely used in nanotechnology, because on their basis many nanostructures of different symmetry have been created, such as quantum dots and their arrays, quantum wires, single and multiple quantum well structures [1-4]. A separate important area is the creation and studies of the multilayer plane nanosystems - the so-called resonant tunneling structures (RTS), which are practically used as precision elements of quantum cascade lasers (QCL) [5] and detectors (QCD) [6] etc.

For RTS created on the basis of Group III Nitrides an important role is played by the effects of the potential profile deformation of such nanostructures by an internal electric field, which is a consequence of the arising spontaneous and piezoelectric polarizations [7-10]. Regarding the influence of the potential associated with the piezoelectric effect, such related problem was solved only numerically [11], using the finite difference method in order to study the thermal conductivity of a single-layer GaN nanofilm. The spectrum of acoustic phonons with allowance for the piezoelectric effect was not investigated itself; only the group velocities of acoustic phonons were calculated. It should be noted that the approach proposed in this paper is not applicable for nanosystems with a large number of layers due to the used boundary conditions. Firstly, these are zero conditions for the stress tensor components at the boundary with the external environment, and secondly, the boundary conditions for the components of the elastic displacement vector are not provided or are not used at all.

Besides, it is necessary to highlight a group of papers considering the studies of the piezoelectric effect and acoustic waves propagation in piezoelectric semiconductor crystals and nanostructures [12-17], furthermore, part of them were performed for the nitride-based semiconductors [12-14, 16].

The interaction of electrons with acoustic phonons in nitride-based RTS has been investigated recently [18]. However, the interaction of electrons with acoustic phonons via the piezoelectric potential for nitride semiconductor nanostructures generally remains unexplored, moreover, for the case of interaction via the deformation potential the influence of the piezoelectric effect has not been investigated either. Similar problem for a GaAs quantum well was considered in the paper [19]. In this paper the piezoelectric potential was calculated in the first order of the perturbation theory, neglecting the effects of the piezoelectric field on the components of the elastic displacement field for acoustic phonons. Direct numerical calculations in this paper were not performed. So, it should be concluded that theoretical studies of

\footnotetext{
*Corresponding author

(1. boyko.i.v.theory@gmail.com (I. Boyko); Mykhaylo_Petryk@tntu.edu.ua (M. Petryk);

jacques.fraissard@sorbonne-universite.fr (J. Fraissard)

ORCID(s): 0000-0003-2787-1845 (I. Boyko); 0000-0001-6612-7213 (M. Petryk); 0000-0002-9465-1933 (J. Fraissard)
} 
the piezoelectric effect influence on electronic processes associated with acoustic phonons are not available now and are of another new problem from the point of view of theory and mathematical formulation relatively similar papers [18-23].

In the proposed paper exact analytical solutions of the differential equations of motion, which describe the elastic displacement of a multilayer nanostructure medium, taking into account the piezoelectric effect, are obtained. The partial components of the Hamiltonian for the electron-acoustic phonons system, which describe their interaction via the piezoelectric potential and deformation potentials, are determined. The spectrum of electrons renormalized by their interaction with acoustic phonons via the piezoelectric and deformation potential was obtained using the method of temperature Green functions. Using the parameters of the cascade of an experimentally created quantum cascade detector as an example, the spectrum of acoustic phonons and electrons are calculated. The calculations of the renormalized electron spectrum and the decay rates of electronic states were performed at the cryogenic and at the room temperature.

\section{Theory of acoustic phonon modes taking into account the piezoelectric effect}

We will study acoustic phonons in a multilayer nanostructure, the geometric shape of which in the Cartesian coordinate system $O x y$ looks like in Fig. 1a. The nanostructure layers corresponding to the quantum wells are the GaN semiconductor, and the layers are potential barriers of the AlN semiconductor. In general, the acoustic phonon modes are determined by finding solutions of the equation of motion for the nanosystem elastic medium, which looks like:

$$
\rho(z) \frac{\partial^{2} u_{i}(\bar{r}, t)}{\partial t^{2}}=\frac{\partial \sigma_{i k}(\bar{r}, t)}{\partial x_{k}} ; x_{1}=x ; x_{2}=y ; x_{3}=z ; i, k=(1 ; 2 ; 3)
$$

where $u_{i}(\bar{r}, t)=u_{i}(x, y, z, t)$ - is the vector of the elastic displacement field corresponding to the point $r=\left(x_{1}, x_{2}, x_{3}\right)=$ $(x, y, z)$, at the moment $t$.

Due to the layered AlN/GaN nature, the density of the nanosystem semiconductor material is dependent on the coordinate $z$, i.e:

$$
\rho=\rho(z)=\sum_{p=0}^{N+1} \rho^{(p)}\left[\theta\left(z-z_{p-1}\right)-\theta\left(z-z_{p}\right)\right], \quad \rho^{(p)}=\left\{\begin{array}{l}
\rho_{0}=\rho_{\mathrm{AlN}}, p-\text { even } \\
\rho_{1}=\rho_{\mathrm{GaN}}, \quad p-\text { odd }
\end{array}, z_{-1}=-\infty ; z_{N+1}=+\infty\right.
$$

- where $\rho^{(p)}$ - is the nanosystem $p$-th layer material density $\left(\rho_{\text {AlN }}\right.$ and $\rho_{\text {GaN }}$ correspondingly are the AlN and GaN semiconductors density), $\theta(z)$ - is the Heaviside unit step function.

Taking into account the influence of the piezoelectric effect we represent the stress tensor as follows:

$$
\sigma_{i k}(\bar{r})=C_{i k l m}(z) u_{l m}(\bar{r})-e_{i k l}(z) E_{l}(\bar{r}), l, m=(1 ; 2 ; 3)
$$

where the second term in the relation (3) is responsible for the piezoelectric effect with an electric field strength $E_{l}(\bar{r})$. It is obvious, that the elastic $C_{i k l m}(z)$ and piezoelectric constants $e_{i k l}(z)$, the dielectric tensor components $\varepsilon_{i k}(\omega, z)$ also depend on the coordinate $z$ as it follows:

$$
\left(C_{i k l m}(z) e_{i k l}(z) \varepsilon_{i k}(\omega, z)\right)^{T}=\sum_{p=1}^{N+1}\left(C_{i k l m}^{(p)} e_{i k l}^{(p)} \varepsilon_{i k}^{(p)}(\omega)\right)^{T}\left[\theta\left(z-z_{p-1}\right)-\theta\left(z-z_{p}\right)\right]
$$

where $C_{i k l m}^{(p)}$ and $e_{i k l}^{(p)}, \varepsilon_{i k}^{(p)}(\omega)$ - are their corresponding values in the $p$-th nanosystem layer.

The strain tensor components are defined as follows:

$$
u_{l m}(\bar{r})=\frac{1}{2}\left(\frac{\partial u_{l}(\bar{r})}{\partial x_{m}}+\frac{\partial u_{m}(\bar{r})}{\partial x_{l}}\right)
$$

then the equation describing the relation between the electric field and the elastic displacement of the semiconductor medium looks like:

$$
D_{i}(\bar{r}, t)=e_{i l m}(z) u_{l m}(\bar{r})+\varepsilon_{i k}(\omega, z) E_{k}(\bar{r}, t) .
$$



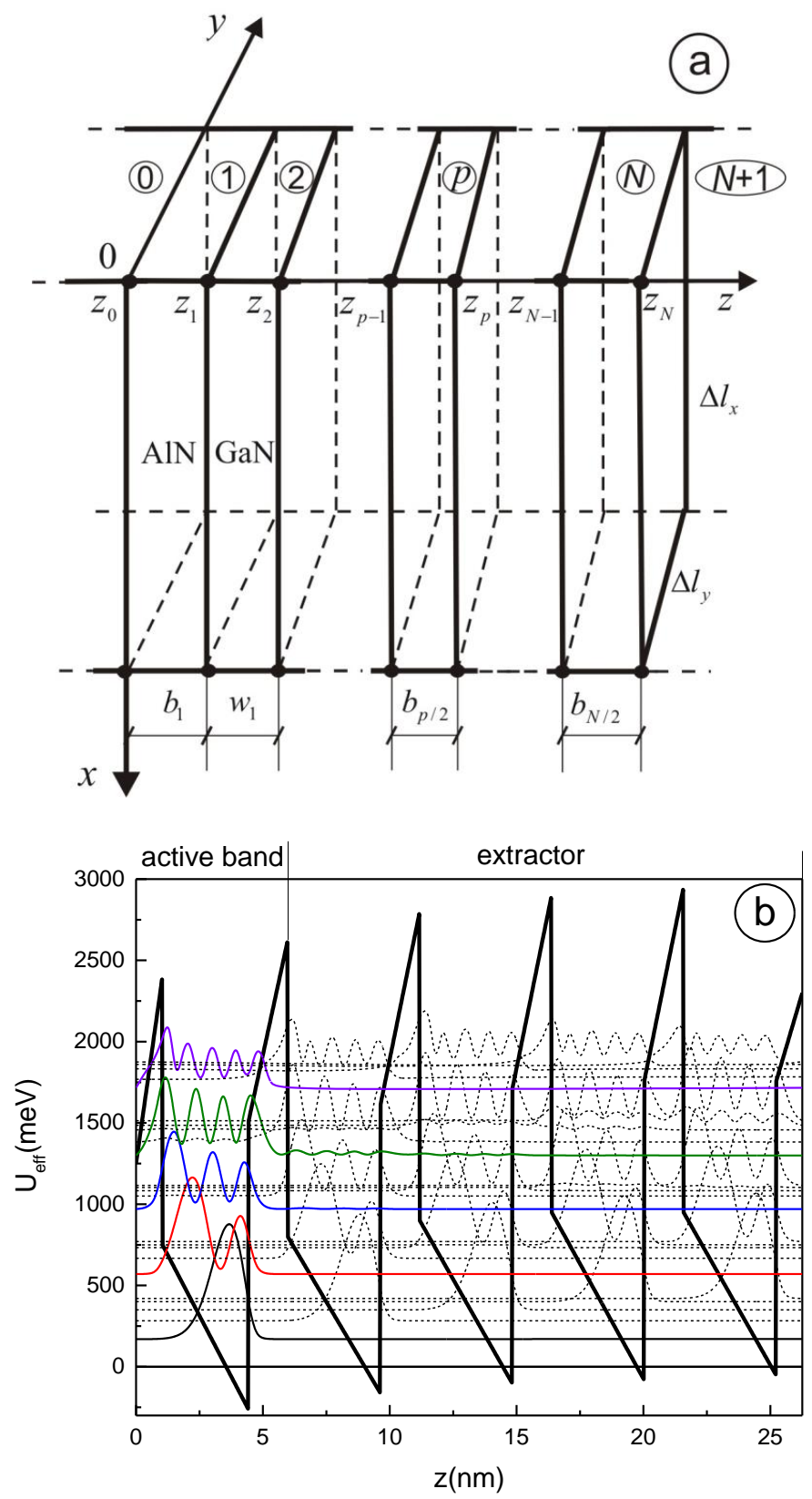

Figure 1: Geometric scheme of the multilayer superlattice (a) and the effective potential of a separate cascade of the quantum cascade detector from the paper [6] with plotted squared moduli of the wave functions for the first $(n=5)$ electronic states localized in each of the quantum well $\left(\left|\Psi_{n}\left(E_{n}, z\right)\right|^{2}\right.$ referring to the corresponding energy values of electronic levels $E_{n}$ ) (b).

It is assumed, that the investigated nanosystem is electrically neutral, then, neglecting the density of free charges $\rho_{\text {ch } \arg \text { es }}=0$, we obtain from the Maxwell's equation:

$$
\operatorname{div} D_{i}(\bar{r}, t)=\left(\bar{\nabla} \cdot D_{i}(\bar{r}, t)\right)=0 .
$$

Further, having assumed, that the components of the elastic displacement and, accordingly, the electric field asso- 
ciated with this displacement have a harmonic dependence on time

$$
u_{i}(\bar{r}, t)=u_{i}(\bar{r}) e^{i \omega t} ; E_{i}(\bar{r}, t)=E_{i}(\bar{r}) e^{i \omega t}
$$

then, the system of coupled equations (1) and (7) taking into account Eq. (3), (5) and (8) looks like:

$$
\left[\begin{array}{c}
\rho(z) \omega^{2} u_{i}(\bar{r}) \delta_{k i}-\frac{\partial}{\partial x_{k}}\left(C_{i k l m}(z) \frac{\partial u_{l}(\bar{r})}{\partial x_{m}}\right)+\frac{\partial}{\partial x_{k}}\left(e_{i k l}(z) E_{l}(\bar{r})\right)=0 ; \\
\frac{\partial}{\partial x_{i}}\left(e_{i k l}(z) \frac{\partial u_{k}(\bar{r})}{\partial x_{l}}\right)+\frac{\partial}{\partial x_{i}}\left(\varepsilon_{i k}(\omega, z) E_{k}(\bar{r})\right)=0 .
\end{array}\right.
$$

Since the investigated RTS is homogeneous relatively the $x O y$ cross-section and acoustic waves propagate along the $O x$ axis, it is necessary to represent the elastic displacement $u_{i}(\bar{r})$ and the electric field $E_{i}(\bar{r})$ strength as a function of $x$ and $z$ :

$$
\begin{aligned}
& V_{i}(\bar{r})=V_{i}(x, z)=\sum_{p=1}^{N+1} V_{i}^{(p)}(x, z)\left[\theta\left(z-z_{p-1}\right)-\theta\left(z-z_{p}\right)\right]=\sum_{p=1}^{N+1} V_{i}^{(p)}(z)\left[\theta\left(z-z_{p-1}\right)-\theta\left(z-z_{p}\right)\right] e^{-i q x} ; \\
& V_{i}=\left\{\begin{array}{lll}
u_{i}, & E_{i}
\end{array}\right\}, u_{i}(z)=\left(\begin{array}{ccc}
u_{1}^{(p)}(z) & u_{2}^{(p)}(z) & u_{3}^{(p)}(z)
\end{array}\right)^{T}, E_{i}^{(p)}(z)=\left(\begin{array}{ccc}
E_{x}^{(p)}(z) & 0 & 0
\end{array}\right)^{T} .
\end{aligned}
$$

where $u_{1}^{(p)}(z), u_{2}^{(p)}(z), u_{3}^{(p)}(z)$ - are the elastic displacement vector components, defined in separate nanosystem $p$-th layer and it is taking into account that the electric field in our case has only one nonzero component.

Now using expression (10), the system of differential equations (9) written for separate $p$-th layer of the nanostructure is obtained as it follows:

$$
\left[\begin{array}{l}
\left(\rho^{(p)} \omega^{2} \delta_{k i}-C_{i k l m}^{(p)} \frac{\partial^{2}}{\partial x_{l} \partial x_{m}}\right) u_{k}(x, z)-e_{i k l}^{(p)} \frac{\partial^{2} \varphi(x, z)}{\partial x_{k} \partial x_{l}}=0 \\
e_{i k l}^{(p)} \frac{\partial^{2} u_{k}(x, z)}{\partial x_{i} \partial x_{l}}-\varepsilon_{i k}^{(p)} \frac{\partial^{2} \varphi(x, z)}{\partial x_{i} \partial x_{k}}=0 .
\end{array}\right.
$$

where it is taken into account, that $\bar{E}_{k}(\bar{r})=-\partial \varphi(\bar{r}) / \partial x_{k}$ and $\varphi(\bar{r}, t)=\varphi(x, z, t)=\varphi(z) e^{i(-q x)}-$ is the piezoelectric potential.

Taking into account that the semiconductor layers of the nanosystem are of wurtzite type crystal lattice, it is convenient to switch to the Voigt two-index notation $C_{i k l m} \rightarrow C_{\alpha \beta}, e_{i k l} \rightarrow e_{\alpha \beta}$ [24]. Then the tensors of elastic and piezoelectric constants, and dielectric permittivity can be represented as follows:

$$
\begin{aligned}
& C_{\alpha \beta}^{(p)}=\left(\begin{array}{cccccc}
C_{11}^{(p)} & C_{12}^{(p)} & C_{13}^{(p)} & 0 & 0 & 0 \\
C_{12}^{(p)} & C_{11}^{(p)} & C_{13}^{(p)} & 0 & 0 & 0 \\
C_{13}^{(p)} & C_{13}^{(p)} & C_{33}^{(p)} & 0 & 0 & 0 \\
0 & 0 & 0 & C_{44}^{(p)} & 0 & 0 \\
0 & 0 & 0 & 0 & C_{44}^{(p)} & 0 \\
0 & 0 & 0 & 0 & 0 & C_{66}^{(p)}
\end{array}\right) ; e_{\alpha \beta}^{(p)}=\left(\begin{array}{cccccc}
0 & 0 & 0 & 0 & e_{15}^{(p)} & 0 \\
0 & 0 & 0 & e_{15}^{(p)} & 0 & 0 \\
e_{31}^{(p)} & e_{31}^{(p)} & e_{33}^{(p)} & 0 & 0 & 0
\end{array}\right) ; \\
& \varepsilon_{\alpha \beta}^{(p)}=\varepsilon_{\alpha \beta}^{(p)}(\omega)=\left(\begin{array}{ccc}
\varepsilon_{11}^{(p)}(\omega) & 0 & 0 \\
0 & \varepsilon_{11}^{(p)}(\omega) & 0 \\
0 & 0 & \varepsilon_{33}^{(p)}(\omega)
\end{array}\right),
\end{aligned}
$$

where dielectric permittivity tensor components are determined in the high-frequency case according to the LiddaneSachs-Teller relation:

$$
\varepsilon_{11}(\omega, z)=\varepsilon_{\infty}(z) \frac{\omega^{2}-\omega_{L O\left(E_{1}\right)}^{2}(z)}{\omega^{2}-\omega_{T O\left(E_{1}\right)}^{2}(z)}, \varepsilon_{33}(\omega, z)=\varepsilon_{\infty}(z) \frac{\omega^{2}-\omega_{L O\left(A_{1}\right)}^{2}(z)}{\omega^{2}-\omega_{T O\left(A_{1}\right)}^{2}(z)}
$$


- $\varepsilon_{\infty}$ is the high frequency dielectric constant, $\omega_{L O}$ and $\omega_{L O}$ - are the frequencies of the longitudinal and transverse modes of optical phonons respectively, defined in the vicinity of a $\Gamma$-point as irreducible representations $A_{1}(z)$ and $E_{1}(x y)[13,24,25]$. Taking into account (10), (12) from the system of equations (11) we obtain:

$$
\begin{gathered}
\left(\begin{array}{cccc}
-C_{44}^{(p)} \frac{d^{2}}{d z^{2}}+q^{2} C_{11}^{(p)} & 0 & i q\left(C_{13}^{(p)}+C_{44}^{(p)}\right) \frac{d}{d z} & i q\left(e_{31}^{(p)}+e_{15}^{(p)}\right) \frac{d}{d z} \\
0 & -C_{44}^{(p)} \frac{d^{2}}{d z^{2}}+q^{2} C_{66}^{(p)} & 0 & 0 \\
i q\left(C_{13}^{(p)}+C_{44}^{(p)}\right) \frac{d}{d z} & 0 & -C_{33}^{(p)} \frac{d^{2}}{d z^{2}}+q^{2} C_{44}^{(p)} & -e_{33}^{(p)} \frac{d^{2}}{d z^{2}}+e_{15}^{(p)} q^{2} \\
0 & 0 & -e_{33}^{(p)} \frac{d^{2}}{d z^{2}}+e_{15}^{(p)} q^{2} & i q\left(e_{31}^{(p)}+e_{15}^{(p)}\right) \frac{d}{d z}+\varepsilon_{33}^{(p)} \frac{d^{2}}{d z^{2}}
\end{array}\right)\left(\begin{array}{c}
u_{1}^{(p)}(z) \\
u_{2}^{(p)}(z) \\
u_{3}^{(p)}(z) \\
\varphi_{x}^{(p)}(z)
\end{array}\right)= \\
=\left(\begin{array}{c}
\rho^{(p)} \omega^{2} u_{1}^{(p)}(z) \\
\rho^{(p)} \omega^{2} u_{2}^{(p)}(z) \\
\rho^{(p)} \omega^{2} u_{3}^{(p)}(z) \\
\varepsilon_{11}^{(p)} q^{2}
\end{array}\right)
\end{gathered}
$$

The system of differential equations in matrix form is equivalent to the following equations:

$$
\begin{aligned}
& -C_{44}^{(p)} \frac{d^{2} u_{1}^{(p)}}{d z^{2}}+i q\left(C_{13}^{(p)}+C_{44}^{(p)}\right) \frac{d u_{3}^{(p)}}{d z}+\left(q^{2} C_{11}^{(p)}-\rho^{(p)} \omega^{2}\right) u_{1}^{(p)}+i q\left(e_{31}^{(p)}+e_{15}^{(p)}\right) \frac{d \varphi^{(p)}}{d z}=0 \\
& -C_{44}^{(p)} \frac{d^{2} u_{2}^{(p)}(z)}{d z^{2}}+\left(q^{2} C_{66}^{(p)}-\rho^{(p)} \omega^{2}\right) u_{2}^{(p)}(z)=0, \\
& -C_{33}^{(p)} \frac{d^{2} u_{3}^{(p)}}{d z^{2}}+i q\left(C_{13}^{(p)}+C_{44}^{(p)}\right) \frac{d u_{1}^{(p)}}{d z}+\left(q^{2} C_{44}^{(p)}-\rho^{(p)} \omega^{2}\right) u_{3}^{(p)}+e_{15}^{(p)} q^{2} \varphi^{(p)}-e_{33}^{(p)} \frac{d^{2} \varphi^{(p)}}{d z^{2}}=0, \\
& i q\left(e_{31}^{(p)}+e_{15}^{(p)}\right) \frac{d \varphi^{(p)}}{d z}+e_{15}^{(p)} q^{2} u_{3}^{(p)}-\varepsilon_{11}^{(p)} q^{2} \varphi^{(p)}-e_{33}^{(p)} \frac{d^{2} u_{3}^{(p)}}{d z^{2}}+\varepsilon_{33}^{(p)} \frac{d^{2} \varphi^{(p)}}{d z^{2}}=0 .
\end{aligned}
$$

The Eq. (16) describes shear acoustic phonons. The properties of their spectrum and the elastic displacement field components have been investigated in details for both single-well [20-23, 26] and multilayer nanostructures [27]. It can be seen from of Eq. (1) that in our case the shear acoustic phonons do not contribute to the induced piezoelectric effect. Therefore, this equation is not mentioned below. The case of the electron- shear acoustic phonon interaction via the piezoelectric potential is a separate problem, which we investigate in the paper [28].

The solution of the self-consistent system of equations (15), (17), (18) can be found exactly in an analytical form, using the method described below.

The system of differential equations (15), (17), (18) describing the displacement field components and the electric field caused by the piezoelectric effect in the separate $p$-th layer of the RTS with the introduction of the following notations:

$$
A^{(p)}=\left(\begin{array}{ccc}
a_{1}^{(p)} & 0 & 0 \\
0 & a_{5}^{(p)} & a_{8}^{(p)} \\
0 & -a_{8}^{(p)} & a_{10}^{(p)}
\end{array}\right) ; B^{(p)}=\left(\begin{array}{ccc}
0 & -i a_{2}^{(p)} & -i a_{4}^{(p)} \\
-i a_{2}^{(p)} & 0 & 0 \\
0 & 0 & i a_{4}^{(p)}
\end{array}\right) ; C^{(p)}=\left(\begin{array}{ccc}
a_{3}^{(p)} & 0 & 0 \\
0 & a_{6}^{(p)} & -a_{7}^{(p)} \\
0 & a_{7}^{(p)} & -a_{9}^{(p)}
\end{array}\right)
$$

where

$$
\begin{aligned}
& a_{1}^{(p)}=C_{44}^{(p)} ; a_{2}^{(p)}=a_{2}^{(p)}(q)=q\left(C_{13}^{(p)}+C_{44}^{(p)}\right) ; a_{3}^{(p)}=a_{3}^{(p)}(q, \omega)=\rho^{(p)} \omega^{2}-q^{2} C_{11}^{(p)} ; \\
& a_{4}^{(p)}=q\left(e_{31}^{(p)}+e_{15}^{(p)}\right) ; a_{5}^{(p)}=C_{33}^{(p)} ; a_{6}^{(p)}=a_{6}^{(p)}(q, \omega)=\rho^{(p)} \omega^{2}-q^{2} C_{44}^{(p)} ; a_{7}^{(p)}=e_{15}^{(p)} q^{2} \\
& a_{8}^{(p)}=e_{33}^{(p)} ; a_{9}^{(p)}=\varepsilon_{11}^{(p)} q^{2} ; a_{10}^{(p)}=\varepsilon_{33}^{(p)} .
\end{aligned}
$$


appears as one the matrix differential equation:

$$
A^{(p)} \frac{d^{2} U^{(p)}}{d z^{2}}+B^{(p)} \frac{d U^{(p)}}{d z}+C^{(p)} U^{(p)}=0 ; U^{(p)}=U^{(p)}(z)=\left(\begin{array}{ccc}
u_{1}^{(p)}(z) & u_{3}^{(p)}(z) & \varphi^{(p)}(z)
\end{array}\right)^{T}
$$

The solutions of the Eq. (21) are found as follows:

$$
U^{(p)}(z)=\alpha^{(p)} e^{\lambda z} ; \alpha^{(p)}=\left(\begin{array}{ccc}
\alpha_{1}^{(p)} & \alpha_{2}^{(p)} & \alpha_{3}^{(p)}
\end{array}\right)^{T} .
$$

Taking into account the relations (21) and (22), the equation is obtained from which the eigenvalues $\lambda^{(p)}$ are found. This is a bicubic equation, which is reduced to a cubic equation using the standard procedure. Its cumbersome solutions are obtained using the Cardano's method. Further, the functions $\alpha^{(p)}$ are found, using the Cayley-Hamilton theorem as follows:

$$
\begin{aligned}
& \alpha_{1, s}^{(p)}=i a_{2}^{(p)} \lambda_{s}^{(p)}=i q\left(C_{13}^{(p)}+C_{44}^{(p)}\right) \lambda_{s}^{(p)} ; \alpha_{2, s}^{(p)}=a_{1}^{(p)}\left(\lambda_{s}^{(p)}\right)^{2}+a_{3}^{(p)}=C_{44}^{(p)}\left(\lambda_{s}^{(p)}\right)^{2}+\rho^{(p)} \omega^{2}-q^{2} C_{11}^{(p)} \\
& \alpha_{3, s}^{(p)}=\frac{\left(a_{2}^{(p)} \lambda_{s}^{(p)}\right)^{2}+\left[a_{1}^{(p)}\left(\lambda_{s}^{(p)}\right)^{2}+a_{3}^{(p)}\right]\left[a_{5}^{(p)}\left(\lambda_{s}^{(p)}\right)^{2}+a_{6}^{(p)}\right]}{-a_{8}^{(p)}\left(\lambda_{s}^{(p)}\right)^{2}+a_{7}^{(p)}}= \\
& =\frac{\left[q\left(C_{13}^{(p)}+C_{44}^{(p)}\right) \lambda_{s}^{(p)}\right]^{2}+\left[C_{44}^{(p)}\left(\lambda_{s}^{(p)}\right)^{2}+\rho^{(p)} \omega^{2}-q^{2} C_{11}^{(p)}\right]\left[C_{33}^{(p)}\left(\lambda_{s}^{(p)}\right)^{2}+\rho^{(p)} \omega^{2}-q^{2} C_{44}^{(p)}\right]}{e_{15}^{(p)} q^{2}-e_{33}^{(p)}\left(\lambda_{s}^{(p)}\right)^{2}}
\end{aligned}
$$

where the norm of a vector $\alpha_{s}^{(p)}$, that is, the $\left\|\alpha_{s}^{(p)}\right\|$ value is found as follows:

$$
\left\|\alpha_{s}^{(p)}\right\|=1 / \sqrt{\left|\alpha_{1, s}^{(p)}\right|^{2}+\left|\alpha_{2, s}^{(p)}\right|^{2}+\left|\alpha_{3, s}^{(p)}\right|^{2}}
$$

So, the final solutions of the system of differential equations (15), (17), (18), taking into account (24), are as follows:

$$
\begin{aligned}
& u_{1}^{(p)}(z)=\sum_{s=1}^{6} \frac{\alpha_{1, s}^{(p)}}{\left\|\alpha_{s}^{(p)}\right\|} A_{s}^{(p)} e^{\lambda_{s}^{(p)} z}=-i q\left(C_{13}^{(p)}+C_{44}^{(p)}\right) \sum_{\tilde{s}=1}^{3} \frac{\lambda_{\tilde{s}}^{(p)}}{\left\|\alpha_{\tilde{s}}^{(p)}\right\|}\left(A_{2 \tilde{s}-1}^{(p)} e_{\tilde{s}}^{\lambda_{\tilde{s}}^{(p)}}-A_{2 \tilde{s}}^{(p)} e^{-\lambda_{\tilde{s}}^{(p)} z}\right) ; \\
& u_{3}^{(p)}(z)=\sum_{s=1}^{6} \frac{\alpha_{2, s}^{(p)}}{\left\|\alpha_{s}^{(p)}\right\|} A_{s}^{(p)} e^{\lambda_{s}^{(p)} z}=-\sum_{\tilde{s}=1}^{3} \frac{\left[C_{44}^{(p)}\left(\lambda_{\tilde{s}}^{(p)}\right)^{2}+\rho^{(p)} \omega^{2}-q^{2} C_{11}^{(p)}\right]}{\left\|\alpha_{\tilde{s}}^{(p)}\right\|}\left(A_{2 \tilde{s}-1}^{(p)} e^{\lambda_{\tilde{s}}^{(p)} z}+A_{2 \tilde{s}}^{(p)} e^{-\lambda_{\tilde{s}}^{(p)} z}\right) ; \\
& \varphi^{(p)}(z)=\sum_{s=1}^{6} \frac{\alpha_{3, s}^{(p)}}{\left\|\alpha_{s}^{(p)}\right\|} A_{s}^{(p)} e^{\lambda_{s}^{(p)} z}= \\
& =\sum_{\tilde{s}=1}^{3} \frac{\left[q\left(C_{13}^{(p)}+C_{44}^{(p)}\right) \lambda_{\tilde{s}}^{(p)}\right]^{2}+\left[C_{44}^{(p)}\left(\lambda_{\tilde{s}}^{(p)}\right)^{2}+\rho^{(p)} \omega^{2}-q^{2} C_{11}^{(p)}\right]\left[C_{33}^{(p)}\left(\lambda_{\tilde{s}}^{(p)}\right)+\rho^{(p)} \omega^{2}-q^{2} C_{44}^{(p)}\right]}{\left[e_{15}^{(p)} q^{2}-e_{33}^{(p)}\left(\lambda_{\tilde{s}}^{(p)}\right)^{2}\right]\left\|\alpha_{\tilde{s}}^{(p)}\right\|} \times \\
& \times\left(A_{2 \tilde{s}-1}^{(p)} e^{\lambda_{\tilde{s}}^{(p)} z}+A_{2 \tilde{s}}^{(p)} e^{-\lambda_{\tilde{s}}^{(p)} z}\right) . \quad
\end{aligned}
$$

These solutions within the studied nanostructure are as follows:

$$
\begin{aligned}
& U(q, \omega, z)=U^{(0)}(q, \omega, z) \theta(-z)+U^{(N+1)}(q, \omega, z) \theta\left(z-z_{N}\right)+\sum_{p=1}^{N} U^{(p)}(q, \omega, z)\left[\theta\left(z-z_{p-1}\right)-\theta\left(z-z_{p}\right)\right], \\
& U=\left\{\begin{array}{lll}
u_{1} & u_{3} & \varphi
\end{array}\right\} .
\end{aligned}
$$


In the relations (26) it was taken into account that, in accordance with the requirement of monotonic decrease for functions $u_{1}(q, \omega, z), u_{3}(q, \omega, z), \varphi(q, \omega, z)$ at $z \rightarrow \pm \infty$, the following condition must be provided:

$$
\lim _{z \rightarrow \pm \infty}\left(u_{1}(q, \omega, z), u_{3}(q, \omega, z), \varphi(q, \omega, z)\right)^{T}=\left(\begin{array}{ccc}
0 & 0 & 0
\end{array}\right)^{T}
$$

from where we obtain: $A_{2}^{(0)}=A_{4}^{(0)}=A_{6}^{(0)}=A_{1}^{(N+1)}=A_{3}^{(N+1)}=A_{5}^{(N+1)}=0$. The rest unknown coefficients are uniquely determined by the boundary conditions for the displacement field components of acoustic phonons $u_{1(3)}^{(p)}(q, \omega, z)$, the components of the stress tensor $\sigma_{x z}(q, \omega, z), \sigma_{z z}(q, \omega, z)$, as well as piezoelectric potential $\varphi^{(p)}(q, \omega, z)$ and normal components of the electric displacement vector $D_{z}^{(p)}(q, \omega, z)$ :

$$
\left[\begin{array}{c}
\left.u_{1(3)}^{(p)}(q, \omega, z)\right|_{z=z_{p}-0}=\left.u_{1(3)}^{(p+1)}(q, \omega, z)\right|_{z=z_{p}+0}, \\
\left.\sigma_{x z}^{(p)}(q, \omega, z)\right|_{z=z_{p}-0}=\left.\sigma_{x z}^{(p+1)}(q, \omega, z)\right|_{z=z_{p}+0}, \\
\left.\sigma_{z z}^{(p)}(q, \omega, z)\right|_{z=z_{p}-0}=\left.\sigma_{z z}^{(p+1)}(q, \omega, z)\right|_{z=z_{p}+0}, \\
\left.\varphi^{(p)}(q, \omega, z)\right|_{z=z_{p}-0}=\left.\varphi^{(p+1)}(q, \omega, z)\right|_{z=z_{p}+0}, \\
\left.D_{z}^{(p)}(q, \omega, z)\right|_{z=z_{p}-0}=\left.D_{z}^{(p+1)}(q, \omega, z)\right|_{z=z_{p}+0},
\end{array}\right.
$$

where

$$
\begin{aligned}
& \sigma_{x z}^{(p)}(q, \omega, z)=\sigma_{13}^{(p)}(q, \omega, z)-e_{15}^{(p)} E_{x}^{(p)}(q, \omega, x, z)=\frac{1}{2} C_{44}^{(p)}\left(\frac{\partial u_{z}^{(p)}(x, z)}{\partial x}+\frac{\partial u_{x}^{(p)}(x, z)}{\partial z}\right)- \\
& -e_{15}^{(p)} \frac{\partial}{\partial x}\left(E_{x}^{(p)}(z) e^{i(\omega t-q x)}\right)=\left[\frac{1}{2} C_{44}^{(p)}\left(-i q u_{3}^{(p)}(z)+\frac{d u_{1}^{(p)}(z)}{d z}\right)+i q e_{15}^{(p)} \frac{d \varphi^{(p)}(z)}{d z}\right] e^{i(\omega t-q x)} ; \\
& \sigma_{z z}^{(p)}(q, \omega, z)=\sigma_{33}^{(p)}(q, \omega, z)=C_{13}^{(p)} \nabla \cdot u_{i}^{(p)}(x, z)+\left(C_{33}^{(p)}-C_{13}^{(p)}\right) \frac{\partial u_{i}^{(p)}(x, z)}{\partial z}= \\
& =\left(-i q C_{13}^{(p)} u_{1}^{(p)}(z)+C_{33}^{(p)} \frac{d u_{3}^{(p)}(z)}{d z}\right) e^{i(\omega t-q x)} ; \\
& D_{z}(z)=e_{31}^{(p)}\left(\frac{\partial u_{x}^{(p)}(x, z)}{\partial x}+\frac{\partial u_{y}^{(p)}(x, z)}{\partial x}\right)+e_{33}^{(p)} \frac{\partial u_{z}^{(p)}(x, z)}{\partial z}=\left(-i q e_{31}^{(p)} u_{1}^{(p)}(z)+e_{33}^{(p)} \frac{d u_{3}^{(p)}(z)}{d z}\right) e^{i(\omega t-q x)} .
\end{aligned}
$$

Using the conditions (28) and for adjacent layers of the nanostructure with indices " $p$ and " $p+1$ " and then applying the transfer matrix method [13, 24, 27], we obtain:

$$
\begin{aligned}
& \left|a^{(p)}\right\rangle=T^{(p, p+1)}(q, \omega)\left|a^{(p+1)}\right\rangle ;\left|a^{(p)}\right\rangle=\left(\begin{array}{llllll}
A_{1}^{(p)} & A_{2}^{(p)} & A_{3}^{(p)} & A_{4}^{(p)} & A_{5}^{(p)} & A_{6}^{(p)}
\end{array}\right)^{T} ; \\
& T^{(p, p+1)}(q, \omega)=\left(t^{(p)}(q, \omega)\right)^{-1} t^{(p+1)}(q, \omega) \text {; } \\
& t_{1,3}^{(p)}=\left(\begin{array}{cccccc}
\alpha_{11}^{(p)} & -\alpha_{11}^{(p)} & \alpha_{12}^{(p)} & -\alpha_{12}^{(p)} & \alpha_{13}^{(p)} & -\alpha_{13}^{(p)} \\
\beta_{11}^{(p)} & \beta_{11}^{(p)} & \beta_{12}^{(p)} & \beta_{12}^{(p)} & \beta_{13}^{(p)} & \beta_{13}^{(p)} \\
\gamma_{11}^{(p)} & \gamma_{11}^{(p)} & \gamma_{12}^{(p)} & \gamma_{12}^{(p)} & \gamma_{13}^{(p)} & \gamma_{13}^{(p)} \\
\delta_{11}^{(p)} & -\delta_{11}^{(p)} & \delta_{12}^{(p)} & -\delta_{12}^{(p)} & \delta_{13}^{(p)} & -\delta_{13}^{(p)} \\
\varsigma_{11}^{(p)} & \varsigma_{11}^{(p)} & \varsigma_{12}^{(p)} & \varsigma_{12}^{(p)} & \varsigma_{13}^{(p)} & \varsigma_{13}^{(p)} \\
\eta_{11}^{(p)} & -\eta_{11}^{(p)} & \eta_{12}^{(p)} & -\eta_{12}^{(p)} & \eta_{13}^{(p)} & -\eta_{13}^{(p)}
\end{array}\right)\left(\begin{array}{c}
e^{\lambda_{1}^{(p)} z_{p}} \\
e^{-\lambda_{1}^{(p)} z_{p}} \\
e^{\lambda_{2}^{(p)} z_{p}} \\
e^{-\lambda_{2}^{(p)} z_{p}} \\
e^{\lambda_{3}^{(p)} z_{p}} \\
e^{-\lambda_{3}^{(p)} z_{p}}
\end{array}\right)
\end{aligned}
$$


where

$$
\begin{aligned}
& \alpha_{1 s}^{(p)}=-i q\left(C_{13}^{(p)}+C_{44}^{(p)}\right) \lambda_{s} /\left\|\alpha_{s}^{(p)}\right\| ; \\
& \beta_{1 s}^{(p)}=-\left(C_{44}^{(p)} \lambda_{s}^{2}+\rho^{(p)} \omega^{2}-q_{11}^{2}(p)\right) /\left\|\alpha_{s}^{(p)}\right\|, \\
& \gamma_{1 s}^{(p)}=\frac{i q}{2\left\|\alpha_{s}^{(p)}\right\|}\left[C_{44}^{(p)}\left(C_{13}^{(p)}+C_{44}^{(p)}\right) \lambda_{s}^{2}-C_{44}^{(p)}\left(\begin{array}{c}
(p) \\
44
\end{array} \lambda_{s}^{2}+\rho^{(p)} \omega^{2}-q^{2} C_{11}^{(p)}\right)+\right. \\
& \left.+\frac{2 e_{15}^{(p)} \lambda_{n}\left(q^{2}\left(C_{13}^{(p)}+C_{44}^{(p)}\right)^{2} \lambda_{s}^{2}\right)+\left(C_{44}^{(p)} \lambda_{s}^{2}+\rho^{(p)} \omega^{2}-q^{2} C_{11}^{(p)}\right)\left(C_{33}^{(p)} \lambda_{s}^{2}+\rho^{(p)} \omega^{2}-q_{44}^{2}(p)\right)}{e_{15}^{(p)} q^{2}-e_{33}^{(p)} \lambda_{s}^{2}}\right], \\
& \delta_{1 s}^{(p)}=-\lambda_{n}\left[\left(C_{13}^{(p)}\left(C_{13}^{(p)}+C_{44}^{(p)}\right)-C_{11}^{(p)} C_{33}^{(p)}\right) q^{2}+C_{33}^{(p)}\left(C_{44}^{(p)} \lambda_{n}^{2}+\rho \omega^{2}\right)\right] /\left\|\alpha_{s}^{(p)}\right\|, \\
& \varsigma_{1 s}^{(p)}=-\frac{q^{2}\left(C_{13}^{(p)}+C_{44}^{(p)}\right)^{2} \lambda_{s}^{2}+\left(C_{44}^{(p)} \lambda_{s}^{2}+\rho^{(p)} \omega^{2}-q^{2} C_{11}^{(p)}\right)\left(C_{33}^{(p)} \lambda_{s}^{2}+\rho^{(p)} \omega^{2}-q^{2} C_{44}^{(p)}\right)}{\left\|\alpha_{s}^{(p)}\right\|\left(e_{15}^{(p)} q^{2}-e_{33}^{(p)} \lambda_{s}^{2}\right)}, \\
& \eta_{1 s}^{(p)}=\frac{\lambda_{n}}{\left\|\alpha_{s}^{(p)}\right\|}\left[\left(C_{13}^{(p)}+C_{44}^{(p)}\right) e_{31}^{(p)} q^{2}-e_{33}^{(p)}\left(C_{44}^{(p)} \lambda_{s}^{2}+\rho^{(p)} \omega^{2}-q^{2} C_{11}^{(p)}\right)\right], \\
& s=1,2,3 .
\end{aligned}
$$

Then, being applied (30) successively for the entire RTS, we obtain its transfer matrix:

$$
\left|a^{(0)}\right\rangle=T(q, \omega)\left|a^{(N+1)}\right\rangle, T(q, \omega)=\prod_{p=1}^{N} T^{(p, p+1)}(q, \omega)
$$

hence, the acoustic phonons spectrum $\Omega_{n_{1}}(q)$ is obtained from such a dispersion equation:

$$
|T(q, \omega)|=\left|\prod_{p=1}^{N}\left(t^{(p)}(q, \omega)\right)^{-1} t^{(p+1)}(q, \omega)\right|=0
$$

Using boundary conditions (28), we express the coefficients $A_{1}^{(p)}, A_{2}^{(p)}, A_{3}^{(p)}, A_{4}^{(p)}, A_{5}^{(p)}, A_{6}^{(p)}$ in terms of one of them. This coefficient is obtained from the normalization condition for the displacement components $u_{1(3)}$, which is as follows:

$$
\int_{-\infty}^{+\infty} \rho(z)\left[u_{1}\left(q, \omega_{q}, z\right) u_{1}^{*}\left(q^{\prime}, \omega_{q^{\prime}}, z\right)+u_{3}\left(q, \omega_{q}, z\right) u_{3}^{*}\left(q^{\prime}, \omega_{q^{\prime}}, z\right)\right] d z=\frac{\hbar}{2 \Delta l_{x} \Delta l_{y} \omega} \delta_{q q^{\prime}},
$$

where $\Delta l_{x} \Delta l_{y}$ - is a cross-sectional area of the nanostructure by plane $x O y$, besides $\Delta l_{x}, \Delta l_{y}>>z_{N}$.

For the components of the elastic displacement $u_{1(3)}\left(q, \omega_{q}, r\right)$, associated with acoustic phonons and the piezoelectric potential $\varphi\left(q, \omega_{q}, r\right)$, the Fourier transform is provided:

$$
U\left(q, \omega_{n_{1} q}, r\right)=\sum_{n_{1} q} U\left(q, \omega_{n_{1} q}, z\right) e^{i q r},
$$

where the functions $u_{1(3)}\left(q, \omega_{n_{1} q}, z\right)$ and $\varphi\left(q, \omega_{n_{1} q}, z\right)$, are the coefficients in the expansion containing the entire frequency spectrum of the flexural and dilatational modes of acoustic phonons $\omega_{n_{1} q}$.

Having performed the known transition from the obtained Fourier components to generalized coordinates and impulses, and then having applied the operators of occupation numbers according to [18, 19, 24], we find the operator for the elastic displacement components in the representation of occupation numbers:

$$
\hat{u}_{1(3)}\left(q, \omega_{n_{1} q}, r\right)=\sum_{p=0}^{N+1} \sum_{n_{1} q} \sqrt{\frac{\hbar}{2 \Delta l_{x} \Delta l_{y} \rho^{(p)} \omega_{n_{1} q}}}\left(\hat{b}_{n_{1}}(-\bar{q})+\hat{b}_{n_{1}}(\bar{q})\right) \hat{w}_{1(3)}^{(p)}\left(q, \omega_{n_{1} q}, z\right) e^{i \bar{q} \bar{r}}\left[\theta\left(z-z_{p-1}\right)-\theta\left(z-z_{p}\right)\right],
$$




$$
\hat{w}_{1(3)}^{(p)}\left(q, \omega_{n_{1} q}, z\right)=\sqrt{\rho^{(p)}} \hat{u}_{1(3)}^{(p)}\left(q, \omega_{n_{1} q}, z\right), z_{-1}=-\infty .
$$

Further, to determine $\varphi\left(q, \omega_{n_{1} q}, z\right)$, we substitute expansion (35) into Eq. (17) taking into account (36). After conversion and simplifications of the equation we obtain:

$$
\begin{aligned}
& \frac{d^{2} \varphi^{(p)}\left(q, \omega_{n_{1} q}, z\right)}{d z^{2}}-\frac{e_{15}^{(p)} q^{2}}{e_{33}^{(p)}} \varphi\left(q, \omega_{n_{1} q}, z\right)=\frac{1}{e_{33}^{(p)}} \sqrt{\frac{\hbar}{2 \Delta l_{x} \Delta l_{y} \rho^{(p)} \omega_{n_{1} q}}} \times \\
& \times\left[-C_{33}^{(p)} \frac{d^{2} \hat{w}_{3}^{(p)}\left(q, \omega_{n_{1} q}, z\right)}{d z^{2}}-q^{2}\left(C_{13}^{(p)}+C_{44}^{(p)}\right) \frac{d \hat{w}_{1}^{(p)}\left(q, \omega_{n_{1} q}, z\right)}{d z}+\left(q^{2} C_{44}^{(p)}-\rho^{(p)} \omega^{2}\right) \hat{w}_{3}^{(p)}\left(q, \omega_{n_{1} q}, z\right)\right]
\end{aligned}
$$

Its solutions in general are:

$$
\begin{aligned}
& \varphi^{(p)}\left(q, \omega_{n_{1} q}, z\right)=A_{n_{1}}^{(p)} e^{-\chi_{n_{1}}^{(p)} z}+B_{n_{1}}^{(p)} e^{\chi_{n_{1}}^{(p)} z}+ \\
& +\frac{1}{2 \chi_{n_{1}}^{(p)} e_{33}^{(p)}} \sqrt{\frac{\hbar}{2 \Delta l_{x} \Delta l_{y} \rho^{(p)} \omega_{n_{1} q}}}\left(e^{-\chi_{n_{1}}^{(p)} z} \int_{0}^{z} e^{\chi_{n_{1}}^{(p)} X} F\left(q, \omega_{n_{1} q}, X\right) d X-e^{\chi_{n_{1}}^{(p)} z} \int_{0}^{z} e^{-\chi_{n_{1}}^{(p)} X} F\left(q, \omega_{n_{1} q}, X\right) d X\right), \\
& F\left(q, \omega_{n_{1} q}, X\right)=-C_{33}^{(p)} \frac{d^{2} \hat{w}_{3}^{(p)}\left(q, \omega_{n_{1} q}, X\right)}{d z^{2}}-q^{2}\left(C_{13}^{(p)}+C_{44}^{(p)}\right) \frac{d \hat{w}_{1}^{(p)}\left(q, \omega_{n_{1} q}, X\right)}{d z}+ \\
& +\left(q^{2} C_{44}^{(p)}-\rho^{(p)} \omega^{2}\right) \hat{w}_{3}^{(p)}\left(q, \omega_{n_{1} q}, X\right), \chi_{n_{1}}^{(p)}=q \sqrt{e_{15}^{(p)} / e_{33}^{(p)}} .
\end{aligned}
$$

where the coefficients $A_{n_{1}}^{(p)}$ and $B_{n_{1}}^{(p)}$ are determined from the last two boundary conditions (21).

Now, the Hamiltonian for acoustic phonons in the second quantization representation is as follows:

$$
\hat{H}_{a c}=\sum_{n_{1} q} \hbar \omega_{n_{1} q}\left(\hat{b}_{n_{1} q}^{+} \hat{b}_{n_{1} q}+\frac{1}{2}\right)
$$

where $\hat{b}_{n_{1} q}^{+}$and $\hat{b}_{n_{1} q}$ - are the operators of creation and annihilation of the phonon state in the Bose representation respectively.

\section{Theory of the electron-acoustic phonon interaction via deformation and piezoelectric potentials in the presence of the piezoelectric effect}

To develop a theory of the electron-acoustic phonon interaction, at first we find the solutions of the electronic problem - wave functions of an electron and its spectrum. In the general case, it is necessary to separate the electrons motion along the $\mathrm{Oz}$ axis from their motion in the $x O y$ plane, which is important for of functioning nanodevices. Taking into account the fact, that the transverse dimensions of the nanostructure are much smaller than its longitudinal dimensions $\left(\Delta l_{x}, \Delta l_{y}>>d, d=b_{1}+w_{1}+\ldots+b_{n}\right)$, it makes possible to represent the electron wave function due to the papers $[18,29]$ as follows:

$$
\Psi_{E k}(r, z)=\frac{1}{\sqrt{\Delta x \Delta y}} e^{i \bar{k} \bar{r}} \Psi_{E}(z)
$$

where $\bar{k}$ - is the electron quasimomentum, $\bar{r}$ - is the vector in plane $x O y$.

So, in this case we can already investigate the electronic states of the nanostructure looking for solutions of the Schrodinger and Poisson system of equations:

$$
\left\{\begin{array}{l}
{\left[-\frac{\hbar^{2}}{2} \frac{d}{d z} \frac{1}{m(z)} \frac{d}{d z}+V_{\mathrm{eff}}(z)-E_{n \bar{k}}+\frac{h^{2} k^{2}}{2 m^{*}}\right] \Psi(z)=0} \\
\frac{d}{d z}\left[\varepsilon_{\infty}(z) \frac{d V_{H}(z)}{d z}\right]=-\frac{e}{\varepsilon_{0}}\left\{e\left[N_{D}^{+}(z)-n(z)\right]+\sum_{p=1}^{N} \sigma_{p}\left(z_{p}\right) \delta\left(z-z_{p}\right)\right\}
\end{array}\right.
$$


where $E_{n \bar{k}}$ - is the total energy of an electron in the nanostructure, and

$$
m_{n}^{*}=\left[\int_{-\infty}^{+\infty}\left(\left|\Psi_{n}(z)\right|^{2} / m(z)\right) d z\right]^{-1}, \Psi_{n}(z)=\left.\Psi_{E k}(r, z)\right|_{k=0}
$$

is the electron effective mass, determined for each $n$-th level of the electronic spectrum, in the calculation of which the contributions from the all the RTS layers $[18,29]$ are taken into account, thus:

$$
m=m(z)=\sum_{p=0}^{N+1} m^{(p)}\left[\theta\left(z-z_{p-1}\right)-\theta\left(z-z_{p}\right)\right], \quad m^{(p)}=\left\{\begin{array}{l}
m_{0}=m_{\mathrm{AlN}}, p-\text { even } \\
m_{1}=m_{\mathrm{GaN}}, p-\text { odd }
\end{array}, z_{-1}=-\infty ; z_{N+1}=+\infty\right.
$$

- where $m^{(p)}$ - is the material density of the $p$-th nanosystem layer $\left(m_{\mathrm{AlN}}\right.$ amd $\left.m_{\mathrm{GaN}}\right)$ - respectively, the effective electron mass for semiconductors AlN and GaN).

To find self-consistent solutions of the system (41) is it necessary to consider the contribution of the effective potential components of the nanostructure for an electron:

$$
V_{\text {eff }}(z)=\Delta E_{C}(z)+V_{H}(z)+V_{H L}(z)+V_{E}(z) .
$$

The physical notion of each of the terms (44) is described in details in the papers [30,31]. $\Delta E_{C}(z)=\Delta E_{C}(z, T)$ - is the energy scheme of the nanostructure for an electron in the model of rectangular potential wells and barriers, which is calculated according to the Varshni relation [30] for a given temperature value $\mathrm{T} ; V_{H}(z)$ - is the Hartree potential determined by the ionized acceptor impurities with a concentration $N_{D}^{+}(z)$ and static charge of electrons with a concentration $n(z)=\left(m(z) k_{B} T / \pi \hbar^{2}\right) \sum_{n}\left|\Psi\left(E_{n}, z\right)\right|^{2} \ln \left|1+\exp \left(E_{F}-E_{n} / k_{B} T\right)\right|$, where $E_{F}-$ is the Fermi level of the nanosystem material, obtained from the neutrality condition, $E_{n}$ - is the $n$-th electronic level energy value; $V_{H L^{-}}$is the exchange-correlation potential in the Hedin-Lundqvist representation, $V_{E}$ - is the potential, which is determined by contribution of the internal electric field $F_{p}=\sum_{k=1 ; k \neq p}^{N}\left(P_{k}-P_{p}\right)\left(z_{k}-z_{k+1}\right) / \varepsilon^{(k)} / \varepsilon^{(p)} \sum_{k=1}^{N}\left(z_{k}-z_{k+1}\right) / \varepsilon^{(k)}[8,9,30,31]$ arising in the $p$-th separate nanostructure layer, caused by the total value of the piezoelectric and spontaneous polarizations $P^{(p)}=P_{p z}^{(p)}+P_{s p}^{(p)}$ $[8,9]$. Besides, in the Poisson equation from system (41), the value $\sigma_{p}\left(z_{p}\right)=\left.P_{p+1}(z)\right|_{z \rightarrow z_{p}}-\left.P_{p}(z)\right|_{z \rightarrow z_{p}}$ - specifies the surface charges arising at the boundaries of the nanosystem layers due to different values of the total polarization in them. The methodology for finding solutions of the system (41) is well known from [30,31] and similar papers. In this regard, we will not describe this issue in details, determining the solutions of the system (41) with the required accuracy, using the finite difference method [30] or the semi-analytical method [31]. Then we approximate the nanostructure effective potential for an electron (37), representing it as a piecewise continuous function:

$$
U_{\text {appr }}(z)=\sum_{p=1}^{N} \sum_{l=0}^{M} e F_{\text {eff }}\left(z_{p_{l}}\right) z\left[\theta\left(z-z_{p_{l}}\right)-\theta\left(z-z_{p_{l+1}}\right)\right]
$$

where the strength of an electric field corresponding to the approximated RTS potential is defined as follows:

$$
F_{\text {eff }}\left(z_{p_{l}}\right)=\left(V_{\text {eff }}\left(z_{p_{l+1}}\right)-V_{\text {eff }}\left(z_{p_{l}}\right)\right) / e\left(z_{p_{l+1}}-z_{p_{l}}\right)
$$

where the coordinates of the splitting points of the nanostructure $p$-th layer are: $z_{p_{l}}=l\left(z_{p}-z_{p-1}\right) / 2 M, p=1 \ldots N, z_{0}=0, M$ is the number of these partitions. Taking into account (45) and (46), the solutions of the Schrödinger equation in system (41) are linear combinations of the Airy functions $\mathrm{Ai}(z)$ and $\mathrm{Bi}(z)$ inside of the nanostructure, and decreasing exponents in the external medium to the left and right of it. In general, the wave function of an electron can be represented as follows:

$$
\begin{aligned}
& \Psi_{E k}(E, z)=A^{(0)} e^{\chi^{(0)} z} \theta(-z)+\sum_{p=1}^{N} \sum_{l=0}^{M}\left[A^{\left(p_{l}\right)} A i\left(\zeta^{\left(p_{l}\right)}(z)\right)+B^{\left(p_{l}\right)} B i\left(\zeta^{\left(p_{l}\right)}(z)\right)\right]\left[\theta\left(z-z_{p_{l}}\right)-\theta\left(z-z_{p_{l+1}}\right)\right]+ \\
& +B^{(N+1)} e^{-\chi^{(N+1)} z} \theta\left(z-z_{N+1}\right), \zeta^{\left(p_{l}\right)}(z)=\left(2 m^{\left(p_{l}\right)} e F\left(z_{p_{l}}\right) / \hbar^{2}\right)^{1 / 3}\left[\left(\Delta E_{C}(z)-E_{n \bar{k}}+\frac{h^{2} k^{2}}{2 m^{*}}\right) / e F\left(z_{p_{l}}\right)-z\right], \\
& \chi^{(0)}=\chi^{(N+1)}=\sqrt{2 m_{1}\left(\Delta E_{C}(z)-E_{n \bar{k}}+\frac{h^{2} k^{2}}{2 m^{*}}\right)} / \hbar .
\end{aligned}
$$


For the wave function $\Psi_{E k}(E, z)$ and its density of current continuity at the boundaries of the RTS layers, the boundary conditions being provided:

$$
\Psi_{E k}^{(p)}\left(E_{n \bar{k}}, z_{p}\right)=\Psi_{E k}^{(p+1)}\left(E_{n \bar{k}}, z_{p}\right) ;\left.\frac{d \Psi_{E k}^{(p)}\left(E_{n \bar{k}}, z\right)}{m(z) d z}\right|_{z=z_{p}-\varepsilon}=\left.\frac{d \Psi_{E k}^{(p+1)}\left(E_{n \bar{k}}, z\right)}{m(z) d z}\right|_{z=z_{p}+\varepsilon}, \varepsilon \rightarrow 0
$$

we obtain the dispersion equation for determining the electronic spectrum $E_{n \bar{k}}$. Besides, using boundary conditions (48) and the normalization condition for the wave function $\Psi_{E k}\left(E_{n \bar{k}}, r, z\right)=\Psi_{n k}\left(E_{n \bar{k}}, r, z\right)$, which is presented accordingly to the expression (40):

$$
\int_{-\infty}^{+\infty} \int_{-\infty}^{+\infty} \Psi_{n k}\left(E_{n \bar{k}}, r, z\right) \Psi_{n^{\prime} k^{\prime}}^{*}\left(E_{n^{\prime} \bar{k}^{\prime}}, r, z\right) d r d z=\frac{1}{2 \pi} \delta\left(k-k^{\prime}\right) \delta_{n n^{\prime}}
$$

it is completely determined, since in this way we obtain all coefficients $A^{(0)}, B^{(N+1)}, A^{\left(p_{l}\right)}, B^{\left(p_{l}\right)}$ in solutions (47).

Now for electronic states determined in the RTS effective potential $V_{\text {eff }}(z)$, we represent their wave function using the second quantization method $[18,24]$ as follows:

$$
\hat{\Psi}(r, z)=\sum_{\bar{k}} \sum_{n} \Psi_{n \bar{k}}(\bar{r}, z) \hat{a}_{n \bar{k}}
$$

and then the Hamiltonian of noninteracting electrons takes the well-known appearance [24]:

$$
\hat{H}_{e}=\sum_{n, \bar{k}} E_{n \bar{k}} \hat{a}_{n \bar{k}}^{+} \hat{a}_{n \bar{k}}
$$

where $E_{n \bar{k}}=E_{n}+\hbar^{2} k^{2} / 2 m_{n}^{*}, \hat{a}_{n \bar{k}}^{+}$and $\hat{a}_{n \bar{k}}$-are the creation and annihilation operators of electronic states in the studied nanostructure respectively.

Now let us take into account that in the semiconductors of wurtzite-type lattice the conduction band displacement is defined as follows $[32,33]$ :

$$
\Delta E_{c}=a_{1 c} \varepsilon_{z z}+a_{2 c}\left(\varepsilon_{x x}+\varepsilon_{y y}\right)
$$

where $\varepsilon_{x x}, \varepsilon_{y y}, \varepsilon_{z z}$ - are the stress tensor components, $a_{1 c}, a_{2 c}$-are the deformation potential coupling constants for the conduction band.

Now the Hamiltonian describing the interaction with acoustic phonons via the deformation and piezoelectric potentials in image of the second quantization in phonon variables, is determined as follows:

$$
\begin{aligned}
& \hat{H}_{\mathrm{def}}=\sum_{q n_{1}} \sum_{p=0}^{N+1} \sqrt{\frac{\hbar}{2 \rho^{(p)} \omega_{n_{1} q} \Delta l_{x} \Delta l_{y}}}\left(\hat{b}_{n_{1} q}(\bar{q})+\hat{b}_{n_{1} q}^{+}(-\bar{q})\right)\left(i q a_{2 c} u_{1}^{(p)}\left(q, \omega_{n_{1} q}, z\right)+a_{1 c} \frac{\partial u_{3}^{(p)}\left(q, \omega_{n_{1} q}, z\right)}{\partial z}\right) e^{i \bar{q} \bar{r}} \times \\
& \times\left[\theta\left(z-z_{p-1}\right)-\theta\left(z-z_{p}\right)\right] ; \\
& \hat{H}_{p z}=\sum_{q n_{1}} \sum_{p=0}^{N+1} \varphi^{(p)}\left(q, \omega_{n_{1} q}, z\right)\left(\hat{b}_{n_{1} q}(\bar{q})+\hat{b}_{n_{1} q}^{+}(-\bar{q})\right) e^{i \bar{q} \bar{r}}\left[\theta\left(z-z_{p-1}\right)-\theta\left(z-z_{p}\right)\right]
\end{aligned}
$$

where $\omega_{n_{1}}$ - is the frequency of the acoustic phonons spectrum obtained from the dispersion equation (26), and $n_{1}-$ is the branch number of this spectrum. Thus, the interaction of electrons with acoustic phonons via the deformation and piezoelectric potential is determined by the Hamiltonian as follows:

$$
\hat{H}_{e-\operatorname{def}(e-\mathrm{pz})}=\sum_{n, n^{\prime}, n_{1}, \bar{k}, \bar{q}} F_{n k n_{1} n^{\prime}}^{\mathrm{def}(\mathrm{pz})}(q) \hat{a}_{n^{\prime}, \bar{k}+\bar{q}}^{+} \hat{a}_{n \bar{k}}\left[\hat{b}_{n_{1} q}(\bar{q})+\hat{b}_{n_{1} q}^{+}(-\bar{q})\right],
$$

where the binding functions look like:

$$
\begin{aligned}
& F_{n k n_{1} n^{\prime}}^{\mathrm{def}}(q)=\sqrt{\frac{\hbar}{2 \rho^{(p)} \omega_{n_{1}} \Delta l_{x} \Delta l_{y}}} \int_{z_{p-1}}^{z_{p}} \Psi_{n k}^{(p)}\left(E_{n \bar{k}}, z\right)\left(i q a_{2 c} u_{1}^{(p)}\left(q, \omega_{n_{1} q}, z\right)+\right. \\
& \left.+a_{1 c} \frac{\partial u_{3}^{(p)}\left(q, \omega_{n_{1} q}, z\right)}{\partial z}\right) e^{i \bar{q} \bar{r}} \Psi_{n^{\prime} k}^{*(p)}\left(E_{n^{\prime} \bar{k}}, z\right) d z, \\
& F_{n k n_{1} n^{\prime}}^{\mathrm{pz}}(q)=\int_{z_{p-1}}^{z_{p}} \Psi_{n k}^{(p)}\left(E_{n \bar{k}}, z\right) \varphi^{(p)}\left(q, \omega_{n_{1} q}, z\right) e^{i \bar{q} \bar{r}} \Psi_{n^{\prime} k}^{*(p)}\left(E_{n^{\prime} \bar{k}}, z\right) d z .
\end{aligned}
$$


Thus, the Hamiltonian for a system of the electrons with acoustic phonons in the plane RTS, taking into account the presence of the piezoelectric effect, is:

$$
\hat{H}=\hat{H}_{e}+\hat{H}_{a c}+\hat{H}_{e-\mathrm{def}}+\hat{H}_{e-\mathrm{pz}}
$$

Now, to carry out the renormalization the spectrum of electrons due to their interaction with acoustic phonons via the deformation and piezoelectric potentials in the presence of piezoelectric effect, we perform the Fourier transform of the temperature Green's function, directly obtained from the Dyson equation [18]:

$$
G_{n \bar{k}}(\Omega)=1 /\left(\Omega-E_{n \bar{k}}-M_{n \bar{k}}(\Omega)\right)
$$

Using the weak-coupling approximation for the mass operator $M_{n \bar{k}}(\Omega)$ in Eq. (57) we have:

$$
M_{n \bar{k}}^{\mathrm{def}(\mathrm{pz})}(\Omega)=\sum_{q n_{1} n^{\prime}}\left|F_{n k n_{1} n^{\prime}}^{\mathrm{def}(\mathrm{pz})}(q)\right|^{2}\left[\frac{1+v_{n_{1}}}{\Omega-E_{n^{\prime}, \bar{k}-\bar{q}}-\Omega_{n_{1}}+i \eta}+\frac{v_{n_{1}}}{\Omega-E_{n^{\prime}, \bar{k}+\bar{q}}-\Omega_{n_{1}}+i \eta}\right]
$$

where $\Omega_{n_{1}}=\hbar \omega_{n_{1}}$, and the quantity $v_{n_{1}}=\left(e^{\hbar \omega_{n_{1}} / k_{B} T}-1\right)^{-1}$ - are the occupation numbers of the acoustic phonons spectrum levels, where the first term in (51) describes the creation of acoustic phonons, and the second, the annihilation of acoustic phonons. The mass operator represented as (51), with analytic continuation to the imaginary axis at $\eta \rightarrow \pm 0$, describes the one-phonon approximation.

Further, using equations (57) and operator (58), we study the effects of the interaction of the electrons with acoustic phonons on the electronic spectrum via the deformation and piezoelectric potentials. It is advisable to study this effect by calculating the temperature level shifts of the electronic states of the spectrum $\left(\Delta_{n}\right)$ and the corresponding decay rates $\left(\Gamma_{n}\right)$.

In most of the experimentally realized QCD, in the QCD from the recent paper [6] in particular, the parameters of which are used in our further calculations, the longitudinal motion of electrons parallel to the $z$ axis, that is, perpendicular to the nanostructure layers, is functionally important. Thus, in direct calculations, it will be expedient to assume that the transverse quasimomentum $\bar{k}=0$, then we obtain $\Omega=\left.E_{n \bar{k}}\right|_{\bar{k}=0}=E_{n}$. The energy $\tilde{E}_{n}$ of the electronic level renormalized by the interaction with acoustic phonons can be obtained from the pole of the Fourier transform of the Green's function (57) taking into account the expression (58), which is similar to finding the solutions of the dispersion equation:

$$
\Omega-E_{n}-M_{n}(\Omega)=0
$$

since $\tilde{E}_{n}=\Delta_{n}-\Gamma_{n} / 2[18]$, then from here we find:

$$
\begin{aligned}
& \Delta_{n}^{\operatorname{def}(\mathrm{pz})}=\operatorname{Re}_{n}\left(\Omega=E_{n}, \bar{k}=0\right)=\frac{\Delta l_{x} \Delta l_{y}}{(2 \pi)^{2}} \sum_{n_{1}} \mathrm{P} \iint \frac{\left|F_{n n_{1} n^{\prime}}^{\mathrm{def}(\mathrm{pz})}(q)\right|^{2} d q^{2}}{E_{n}-E_{n, \bar{q}}-\Omega_{n_{1}}} \\
& \Gamma_{n}^{\operatorname{def}(\mathrm{pz})}=-2 \operatorname{Im}_{n}\left(\Omega=E_{n}, \bar{k}=0\right)=\frac{\Delta l_{x} \Delta l_{y}}{2 \pi} \sum_{n_{1}} \iint \delta\left(E_{n}-E_{n, \bar{q}}-\Omega_{n_{1}}^{(\mathrm{FL}, \mathrm{DL})}\right)\left|F_{n n_{1} n^{\prime}}^{\mathrm{def}(\mathrm{pz})}(q)\right|^{2} d q^{2},
\end{aligned}
$$

where in the first integral (60) the symbol $P$ denotes that the integral is taken in accordance with via the Cauchy principal value. The energy of renormalized $n$-th electronic level $\tilde{E}_{n}$, taking into account total temperature shift and its decay rate $\Gamma_{n}$ due to the two considered types of interaction with acoustic phonons is as follows:

$$
\tilde{E}_{n}=E_{n}+\Delta_{n}^{\mathrm{def}}+\Delta_{n}^{\mathrm{pz}}, \Gamma_{n}=\Gamma_{n}^{\mathrm{def}}+\Gamma_{n}^{\mathrm{pz}} .
$$

\section{Discussion of the results}

Using the results of the theory of electron-phonon interaction developed above, calculations were performed for the example of geometric and physical parameters (taken from the papers [14, 25, 33, 34]) of the recently experimentally investigated cascade of QCD [6], which can operate from the mid-infrared to visible spectral range of electromagnetic waves. The geometrical parameters of the cascade layers are as follows: potential barriers $\left(\Delta_{i}^{\exp }, i=\overline{1 . .6}\right):(1.04 ; 1.56 ; 1.56 ; 1.56 ; 1.56 ; 1.04) \mathrm{nm}$, potential wells $\left(d_{i}^{\text {exp }}, i=\overline{1 . .5}\right):(3.38 ; 3.64 ; 3.64 ; 3.64 ; 3.64) \mathrm{nm}$. Besides, the input potential well of width $w_{1}=3.38 \mathrm{~nm}$ is $n$-doped with the concentration of charge carriers: $n_{c}=2 \cdot 10^{19} \mathrm{~cm}^{-3}$ as in the cascade of the experimentally investigated QCD [6].

In Fig. $1 b$ the calculated dependencies on the geometric dimensions $z$ for the squared moduli of the wave functions for the first five electronic states, localized in each of the potential wells of the studied RTS, are shown. The dependencies $W_{n}(z)=\left|\Psi_{n}\left(E_{n}, z\right)\right|^{2}$ for the levels localized in the active band of the cascade are shown by solid lines and for the electronic states of the extractor - by dashed lines. All dependencies are reduced to the energy scale, displaying the corresponding nth level energy value $E_{n}^{(s)}$, where $s$ is the number of the potential well. The dependencies $W_{n}(z)$ formed for each number of level $n$ in each of the potential wells $s$ are 


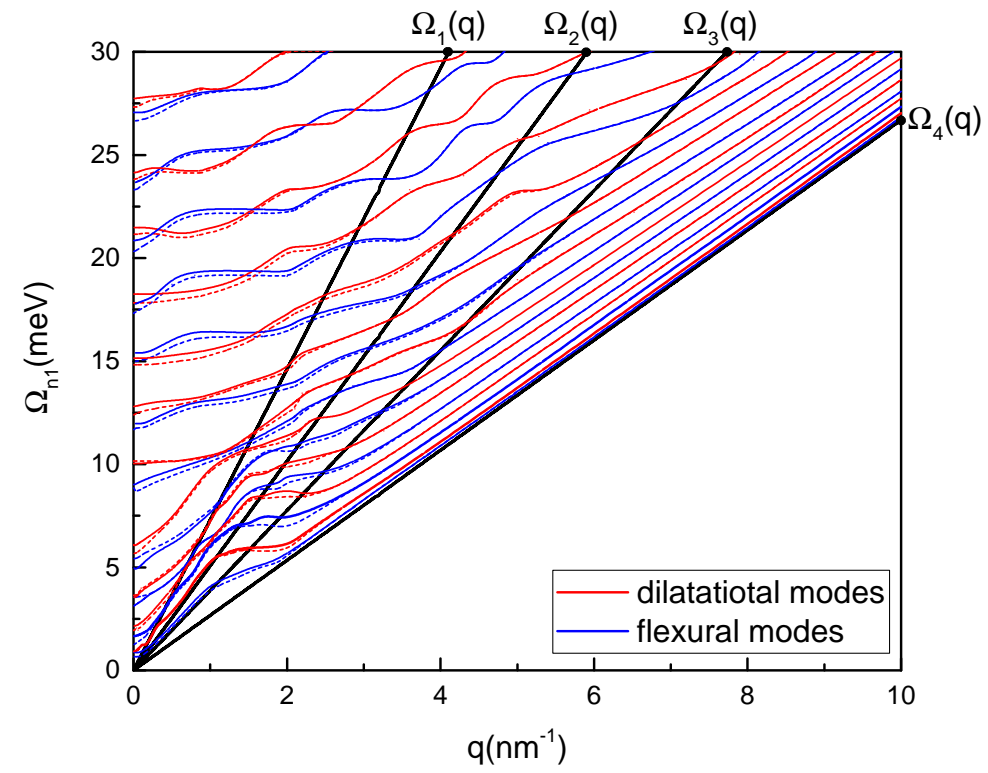

Figure 2: Dependencies of the dilatational (solid and dashed red lines) and flexural (solid and dashed blue lines) modes of the acoustic phonons spectrum $\Omega_{n_{1}}$ on wave vector $q$ as well as the boundaries of the intervals in which they are formed according to the relation (62)

almost identical to each other in terms of localization in the potential well and the $\max W_{n}(z)$ and $\min W_{n}(z)$ values. It can also be seen in Fig. 1b, that for the energy levels the following condition is always provided: $E_{n}^{(s)}<E_{n}^{(s+1)}$, that is, the energy values for levels with the same number $n$ increase in potential wells from left to right.

In Fig. 2 the dependencies on the wave vector $q$ for the flexural and dilatational acoustic phonons branches of the spectrum in the presence of the piezoelectric effect are shown. Similarly, as in the case of the absence of the piezoelectric effect, the spectral dependencies for acoustic phonons are formed within four intervals, however, in this case, the boundaries of these intervals are not determined by the modes of transverse and longitudinal acoustic phonons in bulk AlN and GaN crystals [19-22, 26, 27]. The boundaries of these intervals, which are shown in Fig. 2 with black solid lines, now is obtained from the following expression:

$$
\left[\left(\frac{\tilde{a}^{(p)}(\omega)}{3}\right)^{3}-\frac{\tilde{a}^{(p)}(\omega) \tilde{b}^{(p)}(\omega)}{6}+\frac{\tilde{c}^{(p)}(\omega)}{2}\right]^{2}+\left[\frac{\tilde{b}^{(p)}(\omega)}{3}-\frac{\left(\tilde{a}^{(p)}(\omega)\right)^{2}}{6}\right]^{3}=0
$$

describing the condition for the existence of three real roots of the cubic equation in canonical form with coefficients $\tilde{a}^{(p)}(\omega), \tilde{b}^{(p)}(\omega)$, $\tilde{c}^{(p)}(\omega)$ (no shown due to their cumbersomeness), which results directly from equation (21) Having written the condition (62) for GaN and AlN semiconductors and having numerically found the solutions of (62) for for a given value of $q$, we obtain each time four values $\omega(q)$ for $\mathrm{GaN}$ and AlN. From the obtained values $\omega(q)$, always two values for GaN and AlN satisfy the condition:

$$
\Omega_{a c}^{(\mathrm{GaN}, \mathrm{AlN})}(q) \leq 25-30 \mathrm{meV}
$$

which is known from the papers [21, 27, 34, 35], the other two values for GaN and AlN exceed the values of the acoustic phonon energy in several times, and thus these values can be rejected. So, in decreasing order of the values they acquire depending on the wave number $q$, we get: $\Omega_{1}(q)=\Omega_{1}^{(\mathrm{AlN})}(q) ; \Omega_{2}(q)=\Omega_{2}^{(\mathrm{GaN})}(q) ; \Omega_{3}(q)=\Omega_{3}^{(\mathrm{AIN})}(q) ; \Omega_{4}(q)=\Omega_{4}^{(\mathrm{GaN})}(q)$, where it is indicated, which the semiconductor material corresponds to each of the dependencies shown in Fig. 2 with a black solid lines. It is also clear, that the left value of the first interval is obtained when the value $q=0$, i.e. $\Omega_{n_{1}}^{(0)}=\left.\Omega_{n_{1}}(q)\right|_{q \rightarrow 0}$. In fact, condition (63) is the limit of application of the model of deformation and piezoelectric potentials to study the interaction of electrons with acoustic phonons, since in this case the allowable range of energies of acoustic phonons is only taken into account. In particular, such an application of the deformation potential formalism was effectively used in related papers [21, 22].

As it can be seen from Fig. 2, within the first interval $\left(\Omega_{n_{1}}^{(0)}, \Omega_{1}(q)\right)$ at $q=0$, branches of the acoustic phonons dilatational and flexural modes are formed; their further evolution with a change in $q$ has a number of features, that are special for each of the established intervals. For each type of acoustic phonons, two branches are simultaneously formed, which, with increasing values of the wave vector $q$, tend to merge with each other. The presence of paired branches is especially well seen in the range of the acoustic 

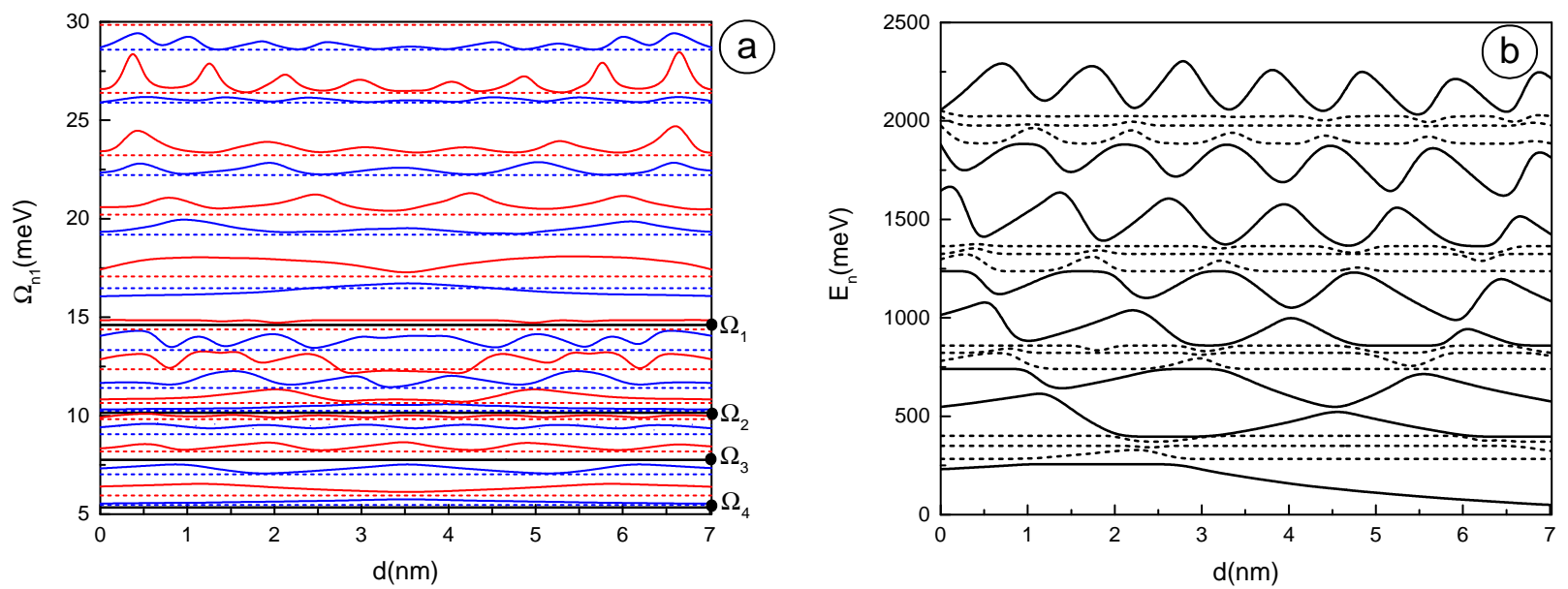

Figure 3: Dependencies of the acoustic phonons spectrum $\Omega_{n_{1}}$ (a) calculated at $q=2 \mathrm{~nm}^{-1}$ and electronic spectrum $E_{n}$ (b) on the relative position of the active band and the extractor in separate cascade $\left(0 \leq d \leq d_{1}+d_{5}\right)$

phonons energies $\Omega_{n_{1}}(q)<10 \mathrm{meV}$, where this effect is, in fact, observed in the range from $\Omega_{n_{1}}^{(0)}$ to values slightly exceeding $\Omega_{3}(q)$ values. The physical meaning of the branches represented by dashed lines will be established by studying the dependence of the spectrum of acoustic phonons on the geometric parameters of the nanostructure. This is due to the fact, that the dependencies on $q$ for the spectrum of both types of phonons are, in fact, the same. It can also be seen that, for the first interval, the branches of dependencies $\Omega_{n_{1}}(q)$ for flexural and dilatational acoustic phonons can approach each other, forming anticrossings, and in the range of values $\Omega_{n_{1}}(q)>15 \mathrm{meV}$ these branches can intersect many times.

The energy dependencies $\Omega_{n_{1}}(q)$ of acoustic phonons in the second interval $\left(\Omega_{1}(q), \Omega_{2}(q)\right)$ are formed by branches that cross the boundary value $\Omega_{1}(q)$ with of $q$ increasing. In this interval, further convergence of the majority of paired branches and the formation of anticrossings between the branches of flexural and dilatational phonons are observed.

The energy dependencies of acoustic phonons $\Omega_{n_{1}}(q)$ in the third interval $\left(\Omega_{2}(q), \Omega_{3}(q)\right)$ are formed by branches that cross the boundary value $\Omega_{2}(q)$ with increasing $q$. In this interval at $\Omega_{n_{1}}(q)<10 \mathrm{meV}$, the difference in the values of the energy of the paired branches is still quite clearly visible. Besides, from Fig. 2 , it can be seen that in this interval at $\Omega_{n_{1}}(q)>10 \mathrm{meV}$, almost all paired branches already merge with each other completely, and directly the energy dependencies $\Omega_{n_{1}}(q)$ become quasilinear.

The dependencies of the acoustic phonons branches energies in the fourth interval $\left(\Omega_{3}(q), \Omega_{4}(q)\right)$ differ significantly from their dependencies in the other three intervals. When the dependencies $\Omega_{n_{1}}(q)$ reach values that coincide with $\Omega_{3}(q)$, the behaviour of both branches of flexural and dilatational acoustic phonons becomes similar. The branches $\Omega_{n_{1}}(q)$ grow with of $q$ increasing, while remaining practically parallel to the $\Omega_{4}(q)$ dependence and being equidistant to each other $\left(\Omega_{n_{1}+1}(q)-\Omega_{n_{1}}(q)=\right.$ const $)$.

Summarizing, we note that the obtained dependencies of the spectrum of dilatational and flexural acoustic phonons $\Omega_{n_{1}}(q)$ can be partially comparable with the spectral dependencies obtained for single-well AlN/AlGaN nanostructures [21, 23]. First of all, this regards the boundaries in which the branches of dependencies are formed, their behavior at small values of the wavenumber $q$, the evolution of the phonon branches in the interval $\left(\Omega_{3}(q), \Omega_{4}(q)\right)$. Complete agreement cannot be expected due to the multilayer nature of the studied nanostructure, the presence of the piezoelectric effect and, as a consequence, the use of a different kind of boundary conditions

An important aspect is the need to study the dependencies of the acoustic phonons spectrum and the electronic spectrum in the investigated nanostructure on its geometric parameters. For a nanosystem, which is, in fact, a separate cascade of QCD, it is advisable to study the dependencies of the spectral parameters of electrons and acoustic phonons not on the width of the potential well of the active band, but on the relative position of the active band and the extractor in the studied cascade. To perform such calculations, the values of the thicknesses for all potential barriers $\left(\Delta_{i}^{\exp }\right)$, as well as the widths of the internal potential wells of the cascade $\left(d_{2}^{\exp }, d_{3}^{\exp }, d_{4}^{\exp }\right)$, are taken to be fixed and equal, respectively, to their experimentally realized values, which are given above. The sum of the widths of the active potential well and the output potential well of the cascade is also considered as constant: $d_{1}^{\exp }+d_{5}^{\exp }$. We will increase the width of the active potential well from 0 to $d_{1}^{\exp }+d_{5}^{\text {exp }}$, and width of the potential well $d_{5}$ is change from $d_{1}^{\text {exp }}+d_{5}^{\exp }$ to 0 . It is clear, that since in the above calculations next condition is provided: $d_{1}+d_{5}=d_{1}^{\exp }+d_{5}^{\exp }$, then at $d_{1}=d_{1}^{\text {exp }}$ experimentally realized the configuration of the cascade is obtained. The results of the acoustic phonons and electrons spectra calculations depending on the values of $d_{1}$ are shown in Fig. 3a, b.

Taking into account the features of the dependencies $\Omega_{n_{1}}(q)$, the calculations of the dependencies of the acoustic phonons spectrum on the value of $d_{1}$ were carried out for such value of the wave number: $q=2 \mathrm{~nm}^{-1}$. This value of $q$ is chosen due to the 

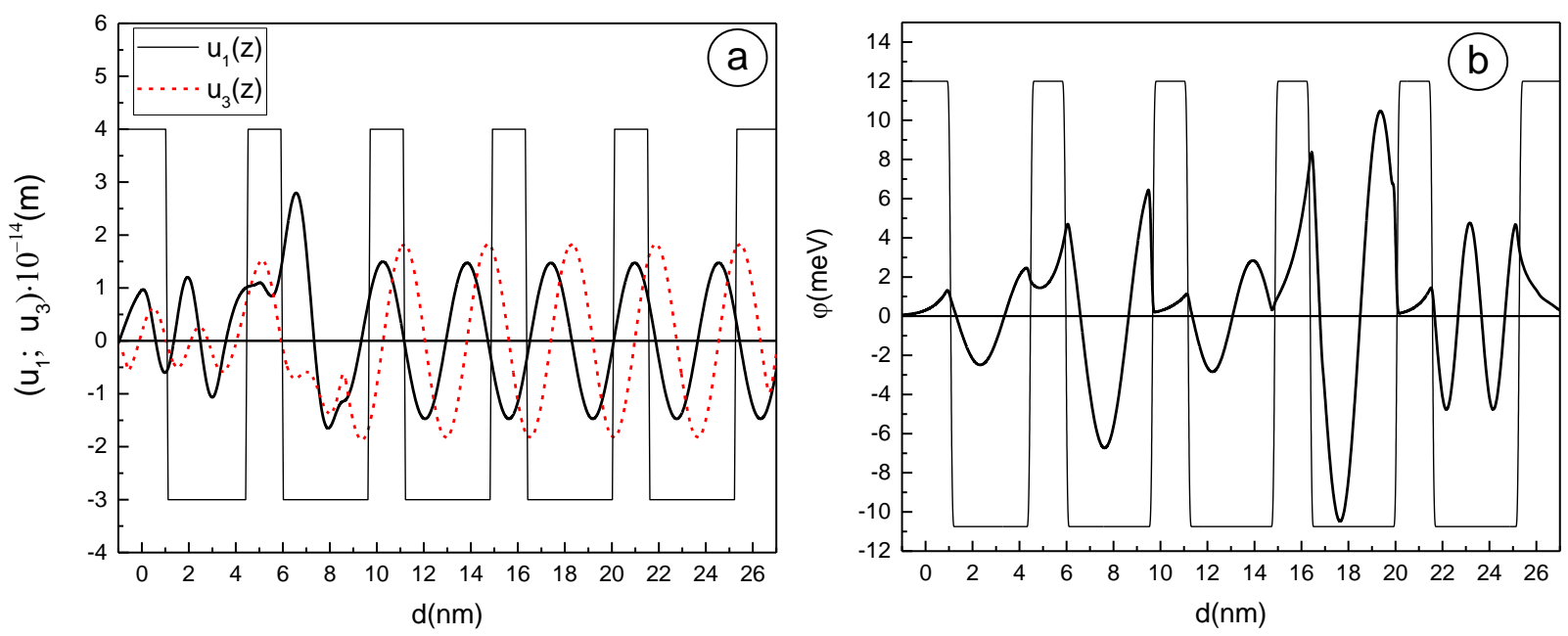

Figure 4: Dependencies of the elastic displacement components $u_{1}(z), u_{3}(z)$ associated with acoustic phonons and the piezoelectric potential $\varphi(z)$ arising in the layers of the nanostructure, calculated at $q=2 \mathrm{~nm}^{-1}$

fact, that for large values of $q$ we would obtain a large number of similar dependencies, and for smaller values of $q$ the contributions of phonon branches from all the intervals established above would not be demonstrated, which is undesirable. As it can be seen from Fig. 3a, the dependencies of the acoustic phonon spectrum $\Omega_{n_{1}}(d)$ are formed within four intervals, the boundaries of which are set above. In each of these intervals branches of the spectrum dependencies are formed and depend both on the value of $d$ and do not depend on it (such branches are shown by dashed lines). It is obvious that the branches that do not depend on $d$ belong to the layers of the nanosystem, the geometric values of which do not change, that is, in fact, they are the contribution of all layers of the cascade without potential wells $d_{1}$ and $d_{5}$. In this case, branches that do not depend on $d$ in all intervals of dependencies are placed mainly between two branches that depend on $d$. The exception is two branches, respectively, of flexural and dilatational acoustic phonons, which intersect with two other branches in their pair; they are located in the first $\left(\Omega \geq \Omega_{1}\right)$ and second $\left(\Omega_{1} \leq \Omega \leq \Omega_{2}\right)$ intervals, respectively. Further we will discuss only the $d$-dependent branches of the phonon spectrum.

For the first interval $\left(\Omega \geq \Omega_{1}\right)$ it is clearly seen, that in this interval the dependencies $\Omega_{n_{1}}(d)$ form $n_{1}$ maxima and $n_{1}-1$ minima, and it should be noted that this effect is also observed for the fourth interval $\left(\Omega_{3} \leq \Omega \leq \Omega_{4}\right)$, but is not observed for the other two intervals. For the second interval $\left.\Omega_{1} \leq \Omega \leq \Omega_{2}\right)$ and for the third interval $\left(\Omega_{2} \leq \Omega \leq \Omega_{3}\right)$, a clear dependence between the number of maxima and minima is not observed: in some branches, the number of minima may be dominated by the number of maxima, in others, their number is equal. It should be noted, that the dependencies $\Omega_{n_{1}}(d)$, that are formed in the range of $d$ change are actually symmetric to the $d \approx\left(d_{1}+d_{5}\right) / 2$ value.

In Fig. $3 \mathrm{~b}$ the dependencies of the electronic spectrum on $d$ values for investigated nanostructure are shown. Solid lines correspond to electronic states localized in the input and output wells of the cascade, dashed lines correspond to electronic states localized in the remaining potential wells. As it can be seen from Fig. 3b, the dependencies $E_{n}(d)$ of the electronic spectrum levels contain both levels, that strongly depend on $d$ and levels, that are relatively slightly dependent on $d$. On the contrary to the case for acoustic phonons, where some of the levels did not demonstrate a dependence on $d$, in the case of an electronic spectrum all levels depend to varying degrees on $d$. For electronic levels localized in the input and output wells of the nanostructure, $n$ maxima and $n-1$ minima are formed, respectively. In this case, due to the fact, that the electronic levels are localized with a nonzero probability in all potential wells of the nanostructure, the formed peculiar anticrossings are always formed by five levels, and are contributed from electronic levels and localized in the $d_{2}, d_{3}, d_{4}$ wells of the cascade, which in the vicinity of these anticrosings demonstrate in fact the most significant dependence on $d$.

In Fig. $4 \mathrm{a}, \mathrm{b}$ the examples of the dependencies for elastic displacement components the $u_{1}(z)$ and $u_{3}(z)$ and the piezoelectric potential $\varphi(z)$ arising in the layers of the nanostructure, which are caused by acoustic phonons, are shown. Presented dependencies were calculated for a fixed value of the wave vector $q$.

As it is seen from Fig. 4a, the components of elastic displacement $u_{1}(z)$ and $u_{3}(z)$ are harmonic functions shifted in phase relatively to each other. In this case, both functions acquire both positive and negative values in the region of the nanostructure and follow to zero in a semiconductor medium outside of it, according to relation (20). As a result, it can be seen, that these functions within separate layer of the nanostructure can behave as symmetric $\left(u_{1}(z)\right)$ and antisymmetric $\left(u_{3}(z)\right)$ functions relatively the center of this layer respectively. It should also be noted, that this effect is not general for all the nanostructure and is most significant in the extractor.

Further, in Fig. 4b the dependencies $\varphi(z)$ for the piezoelectric potential caused by flexural and dilatational acoustic phonons 
Electron-acoustic phonon interaction via the deformation and piezoelectric potentials
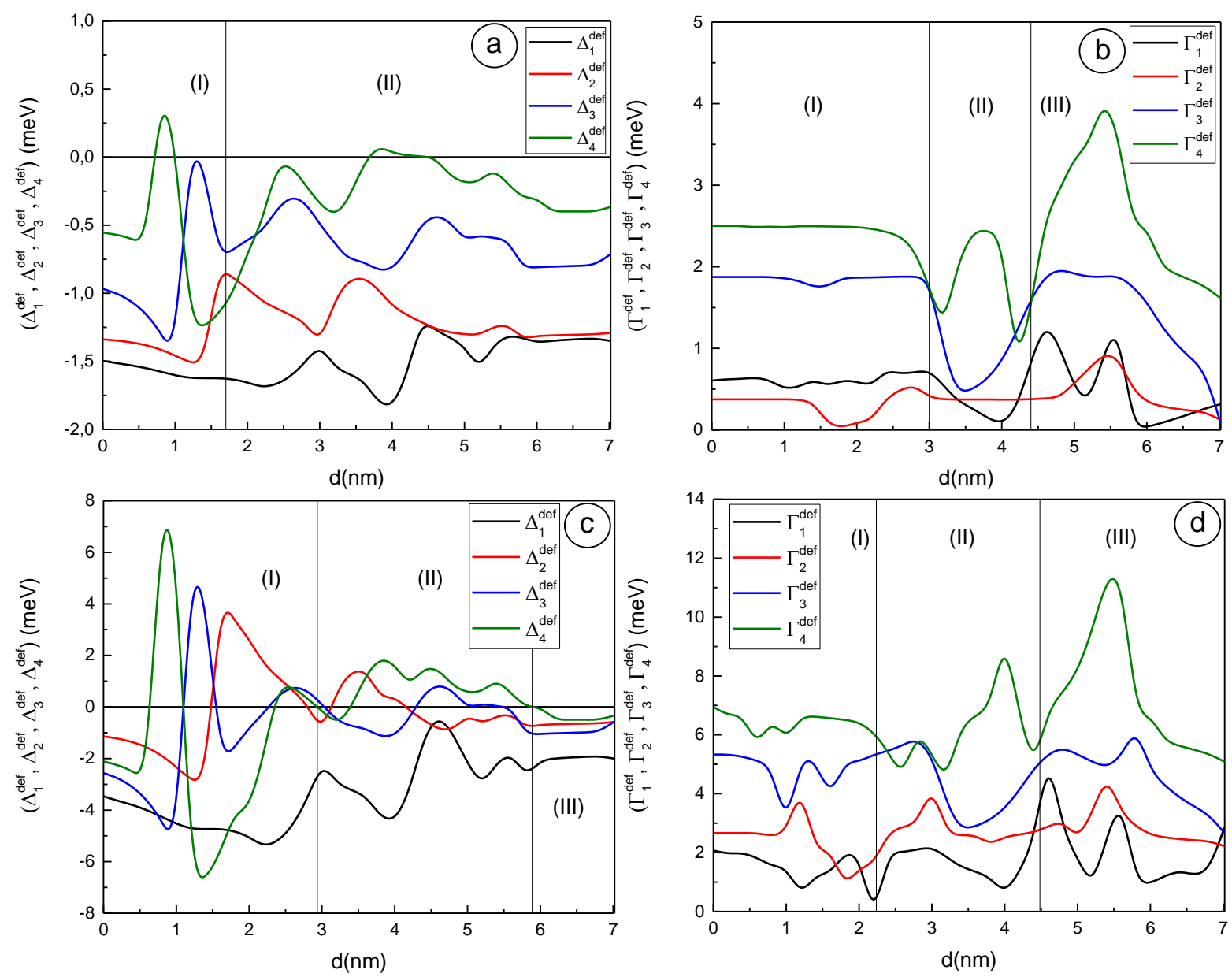

Figure 5: Dependencies of the of the electronic spectrum levels displacements and their decay rates, which are induced by their interaction with dilatational and flexural acoustic phonons via the deformation potential, calculated at $q=2 \mathrm{~nm}^{-1}$ and for temperature values: $\mathrm{T}=77 \mathrm{~K}(\mathrm{a}, \mathrm{b})$ and $\mathrm{T}=300 \mathrm{~K}(\mathrm{c}, \mathrm{d})$

are shown. As it can be seen from the figure, the piezoelectric potential can also take on positive and negative values on the range of $z$, and the absolute maximum values $|\varphi(z)|$ of the function are achieved within the potential wells and at the boundaries with potential barriers. As in the case of displacements $u_{1}(z)$ and $u_{3}(z)$, the piezoelectric potential follows to zero at $z \rightarrow \pm \infty$.

The main and final goal of our investigation is to establish the properties of the interaction of electrons with flexural and dilatational acoustic phonons through the two possible mechanisms, which we have considered: via the deformation potential and via the piezoelectric potential. Since the studied nanostructure was functioning as a separate cascade of QCD, which was experimentally investigated in the paper [6] at liquid nitrogen temperature (77K) and room temperature (300K), we will directly calculate electronic spectrum for the effective potential (44), the temperature shifts for electronic levels and their decay rates exactly at these temperatures. For the purpose of more sufficient visual comparison, the aforementioned characteristics of the electronphonon interaction were calculated depending on the total value of the input and output wells $\left(0 \leq d \leq d_{1}+d_{5}\right)$ as the electronic spectrum in the dependencies in Fig. 3.

In Fig. 5a, b, c, d the dependencies of the temperature shifts of the first four electronic spectrum levels localized in the active band and their decay rates (see (53)) on $d$ of the values are shown, caused by the interaction of electrons with acoustic phonons via the deformation potential at temperatures $\mathrm{T}=77 \mathrm{~K}$ (Fig. 5a, b) and $300 \mathrm{~K}$ (Fig. 5c, d). As it is seen from Fig. 5a, the dependencies $\Delta_{n}^{\mathrm{def}}(d)$ can intersect each other, as it can be seen from region (I) $(0 \mathrm{~nm} \leq d \leq 1.7 \mathrm{~nm})$. However, for dependencies $\Delta_{n}^{\mathrm{def}}(d)$ in general, the following condition is provided: $\Delta_{1}^{\text {def }}(d)>\Delta_{2}^{\text {def }}(d)>\Delta_{3}^{\text {def }}(d)>\Delta_{4}^{\text {def }}(d)$, that is, the lower electron levels shift more, as it can be seen in the region (II) $(1.7 \mathrm{~nm} \leq d \leq 7.02 \mathrm{~nm})$. It should be noted, that the dependencies of the temperature shifts $\Delta_{n}^{\text {def }}(d)$ have a negative sign over the entire range of $d$, except for small intervals in the vicinity of points $d \approx 1 \mathrm{~nm}$ and $d \approx 4 \mathrm{~nm}$ in (I) (II) regions, respectively, for which: $\Delta_{4}^{\text {def }}(d)>0$. The dependencies of the shifts $\Delta_{n}^{\text {def }}(d)$, which were calculated at a temperature 
300K, which are shown in Fig. 5c, differ significantly from the dependencies shown in Fig. 5a. As it can be seen from this figure, the extrema that took place at $\mathrm{T}=77 \mathrm{~K}$ become more pronounced, the absolute values of the shifts increase several times. In this case, the shift of the first electronic level over the entire range of $d$ is negative $\Delta_{1}^{\text {def }}(d)<0$. The dependencies $\Delta_{2}^{\text {def }}(d), \Delta_{3}^{\text {def }}(d), \Delta_{4}^{\text {def }}(d)$ become more symmetric near the abscissa axis, while in the region (I) ( $0 \mathrm{~nm} \leq d \leq 2.9 \mathrm{~nm})$, the maximum, both positive and negative, these displacements are formed. The positions of these extrema on the scale of $d$ values practically coincide with their positions on the dependencies calculated at $\mathrm{T}=77 \mathrm{~K}$. In the region (II) $(2.9 \mathrm{~nm} \leq d \leq 5.8 \mathrm{~nm})$ the shifts $\Delta_{2}^{\text {def }}(d), \Delta_{3}^{\text {def }}(d)$ also form intervals with a positive level shift, wherein the shift $\Delta_{4}^{\text {def }}(d)>0$ in this region. In the region (III) $(5.8 \mathrm{~nm} \leq d \leq 7.02 \mathrm{~nm})$ $\Delta_{n}^{\text {def }}(d)<0$ is obtained .

Further, in Fig. 5b the dependencies on the value of $d$ for the electronic levels decay rates are shown, the calculation of which was carried out at temperature $\mathrm{T}=77 \mathrm{~K}$. As it can be seen from the figure, three different regions can be distinguished in the range of variation of $d$ with characteristic behaviour of $\Gamma_{n}^{\text {def }}(d)$ quantities. Thus, in the region $(\mathrm{I})(0 \mathrm{~nm} \leq d \leq 3 \mathrm{~nm})$, the values of the decay rates $\Gamma_{1}^{\text {def }}(d), \Gamma_{3}^{\text {def }}(d), \Gamma_{4}^{\text {def }}(d)$ depend slightly on $d$, while $\Gamma_{2}^{\text {def }}(d)$ a minima is formed in this region. In region (II) $(3 \mathrm{~nm} \leq d \leq 4.4 \mathrm{~nm})$, the quantity $\Gamma_{2}^{\text {def }}(d)$ depends slightly on $d$, forming minima, $\Gamma_{1}^{\mathrm{def}}(d), \Gamma_{3}^{\mathrm{def}}(d)$, and $\Gamma_{4}^{\mathrm{def}}(d)$ forms two minima and one maximum at the boundary of the region. In the third region (III) $(4.4 \mathrm{~nm} \leq d \leq 7.02 \mathrm{~nm})$, the largest values for all dependencies $\Gamma_{n}^{\text {def }}(d)$ are formed. At the border of this region, the dependencies $\Gamma_{n}^{\text {def }}(d)$ decrease sharply.As it can be seen from Fig. $5 \mathrm{~d}$ the increase of the temperature to $\mathrm{T}=300 \mathrm{~K}$ causes a significant deformation of the dependencies $\Gamma_{n}^{\mathrm{def}}(d)$. The maximum of decay rates values $\Gamma_{n}^{\text {def }}(d)$ is increased significantly from almost 1.5-2 for the first three levels, and more than twice for the fourth electronic level. In region (I) $(0 \mathrm{~nm} \leq d \leq 2.3 \mathrm{~nm})$ all quantities $\Gamma_{n}^{\text {def }}(d)$ have already shown a dependence on $d$, forming pronounced extrema; in region (II) $(2.3 \mathrm{~nm} \leq d \leq 4.3 \mathrm{~nm})$, the dependence $\Gamma_{2}^{n}$ def $(d)$ already forms a clear maximum, extrema of the decay rates dependencies $\Gamma_{1}^{\text {def }}(d), \Gamma_{3}^{\text {def }}(d), \Gamma_{4}^{\text {def }}(d)$, that were formed at $\mathrm{T}=77 \mathrm{~K}$ are now shifted to the left along the abscissa axis. In region (III) $(4.3 \mathrm{~nm} \leq d \leq 7.02 \mathrm{~nm})$, which at $\mathrm{T}=300 \mathrm{~K}$ has, in fact, the same dimensions as at $\mathrm{T}=77 \mathrm{~K}$, the extrema of the dependencies $\Gamma_{n}^{\mathrm{def}}(d)$ do not change their position, in fact, they only increase their values. It should also be noted, that the $\mathrm{T}=300 \mathrm{~K}$ values of the dependencies $\Gamma_{n}^{\text {def }}(d)$ are ordered according to the growth of the number of the electronic level $n\left(\Gamma_{n}^{\text {def }}(d)<\Gamma_{n+1}^{\text {def }}(d)\right)$, except for two small intervals $d$ between regions (I), (II) and (II), (III) for which the following condition is provided: $\Gamma_{1}^{\text {def }}(d)>\Gamma_{2}^{\text {def }}(d)$.

In Fig. 6a, b, c, d the dependencies for the displacements of electronic levels and their decay rates due to interaction with acoustic phonons via the piezoelectric potential are shown (see (53)). As it is seen from Fig. 6a, which shows the dependencies on $d$ for the temperature shifts of the electronic levels at $\mathrm{T}=77 \mathrm{~K}$, the dependencies $\Delta_{n}^{\mathrm{pz}}(d)$ have only a negative sign and have the form of curves that intersect many times. For example, in the first area (I) $(0 \mathrm{~nm} \leq d \leq 2 \mathrm{~nm})$, the maximum and minimum values for $\Delta_{3}^{\mathrm{pz}}(d)$ are formed simultaneously for over the entire range of $d$. At the boundary of this region, the minimum values acquired are $\Delta_{2}^{\mathrm{pz}}(d)$ and $\Delta_{4}^{\mathrm{pz}}(d)$ respectively. In the second region (II) $(2 \mathrm{~nm} \leq d \leq 4.4 \mathrm{~nm})$, it should be taken into account that the values of $\Delta_{1}^{\mathrm{p} z}(d)$ prevail over the values of the attenuation values for other levels, and all dependencies reach their minimum values at the boundaries of this region. In the third region (III) $(4.4 \mathrm{~nm} \leq d \leq 7.02 \mathrm{~nm})$, as it can be seen from the figure, $\Delta_{1}^{\mathrm{pz}}(d)$ and $\Delta_{2}^{\mathrm{pz}}(d)$ slightly depend on $d$, the dependencies $\Delta_{4}^{\mathrm{pz}}(d)$ and $\Delta_{3}^{\mathrm{pz}}(d)$ form one maximum successively.

The influence of the temperature increase on the shifts of the electronic levels is clearly seen from Fig. 6c, which testifies the dependencies $\Delta_{n}^{\mathrm{pz}}(d)$ calculated at the temperature $\mathrm{T}=300 \mathrm{~K}$. As it can be seen from this figure, the temperature increase results in the increase in the absolute values of the shifts $\Delta_{n}^{\mathrm{pz}}(d)$, however, first of all, it should be noted that, they are different from those caused by the electron-phonon interaction via the deformation potential, these shifts have an exclusively negative sign. It should be noted, that with the temperature increase in regions (II) $(2 \mathrm{~nm} \leq d \leq 4.5 \mathrm{~nm})$ and (III) $(4.5 \mathrm{~nm} \leq d \leq 7.02 \mathrm{~nm})$ the condition $\left|\Delta_{n}^{\mathrm{pz}}(d)\right|>\left|\Delta_{n+1}^{\mathrm{pz}}(d)\right|$ is provided. The exception is the first region (I) $(0 \mathrm{~nm} \leq d \leq 2 \mathrm{~nm})$, where the following conditions are realized in narrow ranges of $d:\left|\Delta_{3}^{\mathrm{pz}}(d)\right|>\left|\Delta_{1}^{\mathrm{pz}}(d)\right|$ and $\left|\Delta_{2}^{\mathrm{pz}}(d)\right|>\left|\Delta_{3}^{\mathrm{pz}}(d)\right|$. All the dependencies $\Delta_{n}^{\mathrm{pz}}(d)$ reach minima at the boundaries of these three regions.

Further, in Fig. $6 \mathrm{~b}$ the dependencies $\Gamma_{n}^{\mathrm{pz}}(d)$ for the decay rates of electronic levels at $\mathrm{T}=77 \mathrm{~K}$ are shown. As it can be seen from the figure, the dependencies $\Gamma_{n}^{\mathrm{pz}}(d)$ form three areas, for which the following features can be specified. For region (I) $(0 \mathrm{~nm} \leq d \leq 1.5 \mathrm{~nm})$, from left to right, the decay rates maxima for the fourth, third, and second electronic levels are formed, decay rate of the fist electrons level $\Gamma_{1}^{\mathrm{pz}}(d)$ is slightly dependent on $d$. In the region (II) $(1.5 \mathrm{~nm} \leq d \leq 4.5 \mathrm{~nm}) \Gamma_{2}^{\mathrm{pz}}(d)$ already depend slightly on $d$, and the rest of the dependencies form distinct maxima. In the region (III) ( $4.5 \mathrm{~nm} \leq d \leq 7.02 \mathrm{~nm})$ there are small intervals for which $\Gamma_{2}^{\mathrm{pz}}(d)<\Gamma_{1}^{\mathrm{pz}}(d)$ and $\Gamma_{4}^{\mathrm{pz}}(d)<\Gamma_{3}^{\mathrm{pz}}(d)$. In general, in the whole range of change $d$ we have: $\Gamma_{n}^{\mathrm{pz}}(d)<\Gamma_{n+1}^{\mathrm{pz}}(d)$. The temperature increase to $\mathrm{T}=300 \mathrm{~K}$, as it is seen from Fig. $6 \mathrm{~d}$, causes an increase in the $\Gamma_{n}^{\mathrm{pz}}(d)$ values in several times, while the dependencies $\Gamma_{2}^{\mathrm{pz}}(d)$ and $\Gamma_{3}^{\mathrm{pz}}(d)$ approach each other, being intersected many times. Similarly, as for the dependencies in Fig. $6 \mathrm{~b}$, there are three regions of $d$ variation, the boundaries of which are determined by the almost simultaneous formation of minima of the dependencies $\Gamma_{n}^{\mathrm{pz}}(d)$. In each of these regions, the value of $\Gamma_{4}^{\mathrm{pz}}(d)$ dependence is prevailed by the values of other dependencies. The dimensions of the region (III) $(4.2 \mathrm{~nm} \leq d \leq 7.02 \mathrm{~nm})$ are almost the same as at T=77K. Region (I) $(0 \mathrm{~nm} \leq d \leq 2.2 \mathrm{~nm})$ is larger than at $\mathrm{T}=77 \mathrm{~K}$, and region (II) $(2.2 \mathrm{~nm} \leq d \leq 4.2 \mathrm{~nm})$ is smaller. In all these areas, the extrema of the dependencies $\Gamma_{n}^{\mathrm{pz}}(d)$, which are formed at $\mathrm{T}=77 \mathrm{~K}$, are more sufficient; the formation of additional extrema and some deformation of these dependencies $\Gamma_{n}^{\mathrm{pz}}(d)$ should also be noted.

The calculated energies of quantum transitions between the electronic levels of the active zone, renormalized by the interaction with acoustic phonons due to both mechanisms considered above, are as follows: $\tilde{\Omega}_{n n^{\prime}}=\Omega_{n n^{\prime}}+\Delta_{n}^{\mathrm{def}}+\Delta_{n^{\prime}}^{\mathrm{def}}+\Delta_{n}^{\mathrm{pz}}+\Delta_{n^{\prime}}^{\mathrm{pz}}$. At 

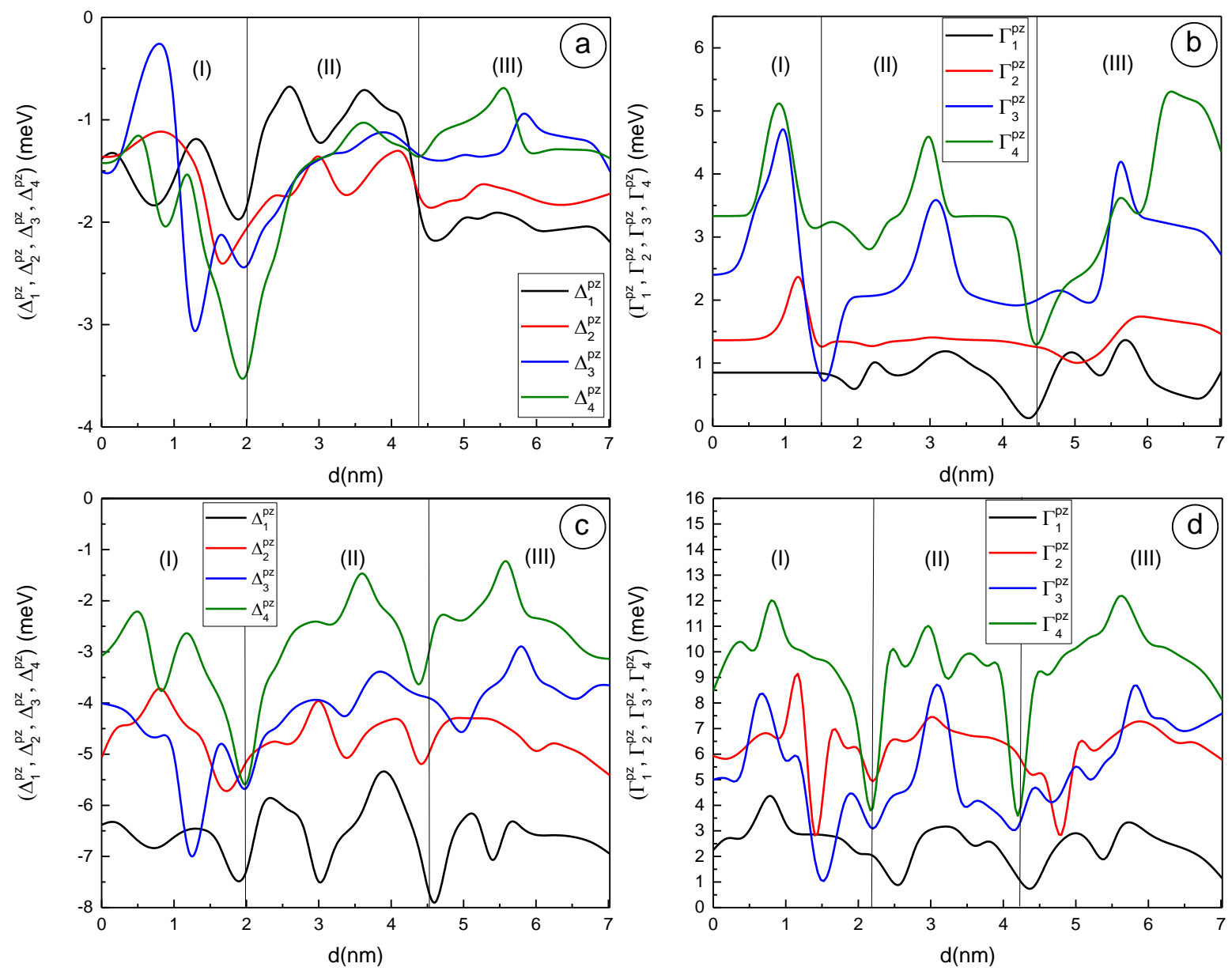

Figure 6: Dependencies of the of the electronic spectrum levels displacements and their decay rates, which are induced by their interaction with dilatational and flexural acoustic phonons via the piezoelectric potential, calculated at $q=2 \mathrm{~nm}^{-1}$ and for temperature values: $\mathrm{T}=77 \mathrm{~K}(\mathrm{a}, \mathrm{b})$ and $\mathrm{T}=300 \mathrm{~K}(\mathrm{c}, \mathrm{d})$

$\mathrm{T}=77 \mathrm{~K}$ it is obtained, that $\tilde{\Omega}_{12}=390.7 \mathrm{meV} ; \tilde{\Omega}_{13}=771.8 \mathrm{meV} ; \tilde{\Omega}_{14}=1039.9 \mathrm{meV}$. At $\mathrm{T}=300 \mathrm{~K}$ it is obtained, that $\tilde{\Omega}_{12}=396.5 \mathrm{meV} ; \tilde{\Omega}_{13}=776.4 \mathrm{meV} ; \tilde{\Omega}_{14}=1047.4 \mathrm{meV}$. These data correlate well with the corresponding values obtained in the paper [6].

Summing up, it should be noted that both mechanisms of interaction of electrons with acoustic phonons with increasing temperature cause rather significant renormalization of the electronic spectrum $\tilde{E}_{n}$ and, accordingly, the energies of electronic transitions $\left(\tilde{\Omega}_{n n^{\prime}}=\tilde{E}_{n^{\prime}}-\tilde{E}_{n}\right)$ and absorption bands $\left(\Gamma_{n n^{\prime}}=\Gamma_{n}+\Gamma_{n^{\prime}}\right)$. An interesting effect obtained is the temperature expansion of the absorption band $\left(\Delta \Gamma_{n n^{\prime}}=\left.\Gamma_{n n^{\prime}}(T)\right|_{T=300 \mathrm{~K}}-\left.\Gamma_{n n^{\prime}}(T)\right|_{T=77 \mathrm{~K}}\right)$. The observed effects directly result in the intensity of quantum transitions between electronic levels and the electronic conductivity of the nanostructure, in fact, decreasing of them due to the increase in the resonance widths of these electronic levels and breaking of the excited electronic states coherence.

\section{Conclusions}

The theory of the flexural and dilatational acoustic phonons spectrum in the presence of piezoelectric effect and the components of elastic displacement caused by these phonons was developed for plane multilayer AlN/GaN nanostructure.

Using the Matsubara temperature Green functions method and the Dyson's equation, an analytical theory of the electron-acoustic phonons interaction via the deformation and piezoelectric potentials at arbitrary temperature values was developed. On the basis of the proposed theory, analytical expressions, that describe the temperature shifts of the electronic spectrum levels and their decay rates were determined, which are direct characteristics of the interaction with acoustic phonons.

Using the physical and geometric parameters of the QCD cascade, which was investigated experimentally, on the basis of the developed theory, the calculations of the dependencies on the wave vector for the spectrum of flexural and dilatational acoustic 
phonons were performed; the dependencies on the geometric design of the nanostructure for the electronic spectrum and the spectrum of flexural and dilatational acoustic phonons, as well as the values of temperature shifts of the electronic spectrum levels and their decay rates were studied.

It is shown that the spectrum of acoustic phonons is formed within four intervals, in which different properties of the spectrum branches appear, both depending on the wave vector and on the geometric parameters of the nanostructure.

It is determined that the temperature shifts of the electronic spectrum levels, caused by the interaction of electrons with acoustic phonons via the deformation potential at temperature values $77 \mathrm{~K}$ and $300 \mathrm{~K}$, acquire both negative and positive values. In this case, the absolute values of the shifts for the electronic levels and their decay rates increase with the temperature increase in several times.

It is found, that the temperature shifts of the electronic levels, caused by the interaction of electrons with acoustic phonons via the piezoelectric potential at temperature values $77 \mathrm{~K}$ and $300 \mathrm{~K}$, acquire only negative values and their absolute values also as well as decay rates of electronic levels are increased with the temperature values increase.

The obtained values of the quantum transitions energies between the renormalized electronic levels are in good agreement with the experimental values.

\section{Acknowledgements}

The authors are grateful to Oleksandr Khimich - Deputy Director of V.M. Glushkov Institute of Cybernetics of National Academy of Sciences of Ukraine for help in organizing and performing cluster computing as well as processing the obtained data

\section{References}

[1] P. A. Crozier, J. Tolle, J. Kouvetakis, C. Ritter, Synthesis of uniform GaN quantum dot arrays via electron nanolithography of $\mathrm{D}_{2} \mathrm{GaN}_{3}$, Appl. Phys. Lett. 84 (2004) 3441. doi : https : //doi .org/10.1063/1.1736314.

[2] B. Amstatt, J. Renard, C. Bougerol, E. Bellet-Amalric, B. Gayral, B. Daudin, Growth of $m$-plane GaN quantum wires and quantum dots on m-plane 6H-SiC, J. Appl. Phys. 102 (2007) 074913. doi: https://doi .org/10.1063/1.2786050.

[3] G. Chen, W. X. Q., K. Fu, x. Rong, H. Hashimoto, B. S. Zhang, et al., Multi-bands photoconductive response in AlGaN/GaN multiple quantum wells, Appl. Phys. Lett. 104 (2014) 172108. doi:https://doi.org/10.1063/1.4874982.

[4] T. Langer, H. Jönen, A. Kruse, H. Bremers, U. Rossow, A. Hangleiter, Strain-induced defects as nonradiative recombination centers in greenemitting GaInN/GaN quantum well structures, Appl. Phys. Lett. 103 (2013) 022108. doi : https://doi.org/10.1063/1.4813446.

[5] V. D. Jovanović, D. Indjin, Z. Ikonić, P. Harrison, Simulation and design of GaN/AlGaN far-infrared ( $\lambda \sim 34 \mu \mathrm{m})$ quantum-cascade laser, Appl. Phys. Lett. 84 (2004) 2995. doi : https://doi.org/10.1063/1.1707219.

[6] P. Qauach, S. F. Liu, A. Jollivet, D. Wang, J. Y. Cheng, N. Isac, et al., A GaN/AlN quantum cascade detector with a broad response from the mid-infrared $(4.1 \mu \mathrm{m})$ to the visible $(550 \mathrm{~nm})$ spectral range, Appl. Phys. Lett. 116 (2020) 171102. doi:https://doi.org/10.1063/5. 0003615.

[7] F. Bernardini, V. Fiorentini, D. Vanderbilt, Spontaneous polarization and piezoelectric constants of III-V nitrides, Phys. Rev. B 56 (1997) R10024(R). doi:https://doi.org/10.1103/PhysRevB.56.R10024.

[8] F. Bernardini, V. Fiorentini, Macroscopic polarization and band offsets at nitride heterojunctions, Phys. Rev. B 57 (1998) R9427(R). doi: https://doi.org/10.1103/PhysRevB.57.R9427.

[9] V. Fiorentini, F. Bernardini, O. Ambacher, Evidence for nonlinear macroscopic polarization in III-V nitride alloy heterostructures, Appl. Phys. Lett. 80 (2002) 1204. doi:https://doi.org/10.1063/1.1448668.

[10] P. Strak, P. Kempisty, K. Sakowski, A. Kaminska, D. Jankowski, K. P. Korona, et al., Ab initio and experimental studies of polarization and polarization related fields in nitrides and nitride structures, AIP Adv. 7 (2017) 015027. doi : https: //doi .org/10.1063/1.4974249.

[11] L. Zhu, H. Luo, On the role of piezoelectricity in phonon properties and thermal conductivity of GaN nanofilms, Theor. Appl. Mech. Lett. 6 (2016) 277-281. doi:https://doi.org/10.1016/j.taml.2016.11.001.

[12] J. Pedrós, F. Calle, J. Grajal, R. J. Jiménez Riobóo, Y. Takagaki, K. H. Ploog, et al., Anisotropy-induced polarization mixture of surface acoustic waves in GaNc -sapphire heterostructures, Phys. Rev. B 72 (2005) 075306. doi: https://doi.org/10.1103/PhysRevB.72.075306.

[13] P. D. Sesion Jr, E. L. Albuquerque, C. Chesman, V. N. Freire, Acoustic phonon transmission spectra in piezoelectric AlN/GaN fibonacci phononic crystals, Eur. Phys. J. B 58 (2007) 379-387. doi: https : //doi .org/10.1140/epjb/e2007-00249-5.

[14] B. K. Sahoo, Effect of piezoelectric polarization on phonon group velocity in nitride wurtzites, J. Mater. Sci. 47 (2011) 2624-2629. doi: https://doi.org/10.1007/s10853-011-6087-2.

[15] M. Lan, P. Wei, Laminated piezoelectric phononic crystal with imperfect interfaces, J. Appl. Phys. 111 (2012) 013505. doi:https://doi. org/10.1063/1.3672404.

[16] X. Huang, C. Du, Y. Zhou, C. Jiang, X. Pu, W. Liu, et al., Piezo-phototronic effect in a quantum well structure, ACS Nano 10 (2016) $5145-5152$. doi:https://doi.org/10.1021/acsnano.6b00417.

[17] B. K. Sahoo, Effect of macroscopic polarization on thermal conductivity of InN, J. Alloys Compd. 605 (2014) 217-221. doi:https: //doi.org/10.1016/j.jallcom.2014.03.168.

[18] I. V. Boyko, M. R. Petryk, Interaction of electrons with acoustic phonons in AlN/ GaN resonant tunnelling nanostructures at different temperatures, Condens. Matter Phys. 605 (2020) 33708. doi: https://doi .org/10.5488/CMP . 23.33708.

[19] N. Bannov, V. Mitin, M. Stroscio, Confined acoustic phonons in a free-standing quantum well and their interaction with electrons, Phys. Status Solidi B 183 (1994) 131-142. doi:https://doi.org/10.1002/pssb.2221830109. 
[20] E. P. Pokatilov, D. L. Nika, A. A. Balandin, Phonon spectrum and group velocities in AlN/GaN/AlN and related heterostructures, Superlattices Microstruct. 33 (2003) 155-171. doi:https://doi.org/10.1016/S0749-6036(03)00069-7.

[21] E. P. Pokatilov, D. L. Nika, A. A. Balandin, Confined electron-confined phonon scattering rates in wurtzite AlN/GaN/AlN heterostructures, J. Appl. Phys. 95 (2004) 5626. doi:https://doi .org/10.1063/1.1710705.

[22] F. J. Yang, S. L. Ban, Acoustic phonon scattering on the two-dimensional electron gas in wurtzite $\mathrm{Al}_{\mathrm{x}} \mathrm{Ga}_{1-\mathrm{x}} \mathrm{N} / \mathrm{GaN} / \mathrm{Al}_{\mathrm{y}} \mathrm{Ga}_{1-\mathrm{y}} \mathrm{N}$ double heterostructures: The ternary mixed crystal effect and size effect, Solid State Commun. 161 (2013) 5-8. doi:https://doi.org/10.1016/ j.ssc. 2013.02.015.

[23] A. Alizadeh, A. Rostami, H. Baghban, H. B. Bahar, Tailoring electron-phonon interaction in nanostructures, Photonics Nanostructures: Fundam. Appl. 12 (2014) 164-172. doi:https://doi.org/10.1016/j.photonics. 2013.11.002.

[24] M. A. Stroscio, M. Dutta, Phonons in Nanostructures, 1st Edition, Cambridge University Press, Cambridge, 2001. doi : https : //doi .org/ 10.1017/CB09780511534898.

[25] J. Piprek, Nitride Semiconductor Devices: Principles and Simulation, 1st Edition, Wiley-VCH Verlag GmbH Co. KGaA, Weinheim, 2007. doi:https://doi.org/10.1002/9783527610723.

[26] F. J. Yang, S. L. Ban, Y. J. Chai, Y. Qu, Acoustic phonon modes in asymmetric quantum wells, Superlattices Microstruct. 102 (2017) $64-73$. doi:https://doi.org/10.1016/j.spmi.2016.12.016.

[27] I. Boyko, M. Petryk, J. Fraissard, Spectrum and normalized modes of acoustic phonons in multilayer nitride-based nanostructure, Eur. Phys. J. B 93 (2020) 57. doi: https://doi .org/10.1140/epjb/e2020-100597-x.

[28] I. Boyko, M. Petryk, J. Fraissard, Theory of the shear acoustic phonons spectrum and their interaction with electrons due to the piezoelectric potential in AlN/GaN nanostructures of plane symmetry, Low Temp. Phys. 47 (2021) 141. doi: https: //doi .org/10.1063/10.0003176.

[29] X. Gao, D. Botez, I. Knezevic, X -valley leakage in GaAs-based midinfrared quantum cascade lasers: A monte carlo study, J. Appl. Phys. 101 (2007) 063101. doi:https://doi.org/10.1063/1.2711153.

[30] S. Saha, J. Kumar, Fully self-consistent analysis of III-nitride quantum cascade structures, J. Comput. Electron. 15 (2016) 1531-1540. doi : https://doi.org/10.1007/s10825-016-0911-5.

[31] I. V. Boyko, Analytical method for calculation of the potential profiles of nitride-based resonance tunneling structures, Condens. Matter Phys. 21 (2018) 43701. doi:https://doi .org/10.5488/CMP. 21.43701.

[32] G. L. Bir, G. E. Pikus, Symmetry and strain-induced effects in semiconductors, John Wiley and Sons, New York, 1974.

[33] Q. Yan, P. Rinke, A. Janotti, M. Scheffler, C. G. Van de Walle, Effects of strain on the band structure of group-III nitrides, Phys. Rev. B 90 (2014) 125118. doi:https://doi.org/10.1103/PhysRevB.90.125118.

[34] I. Vurgaftman, J. R. Meyer, Band parameters for nitrogen-containing semiconductors, J. Appl. Phys. 94 (2003) 3675. doi:https://doi. org/10.1063/1.1600519.

[35] C. Bungaro, K. Rapcewicz, J. Bernholc, Ab initio phonon dispersions of wurtzite aln, gan, and inn, Phys. Rev. B 61 (200) $6720-6725$. doi:https://doi.org/10.1103/PhysRevB.61.6720. 